\title{
The MacMahon Master Theorem for right quantum superalgebras and higher Sugawara operators for $\widehat{\mathfrak{g l}}_{m \mid n}$
}

\author{
A. I. Molev and E. Ragoucy
}

\begin{abstract}
We prove an analogue of the MacMahon Master Theorem for the right quantum superalgebras. In particular, we obtain a new and simple proof of this theorem for the right quantum algebras. In the super case the theorem is then used to construct higher order Sugawara operators for the affine Lie superalgebra $\widehat{\mathfrak{g l}}_{m \mid n}$ in an explicit form. The operators are elements of a completed universal enveloping algebra of $\widehat{\mathfrak{g l}}_{m \mid n}$ at the critical level. They occur as the coefficients in the expansion of a noncommutative Berezinian and as the traces of powers of generator matrices. The same construction yields higher Hamiltonians for the Gaudin model associated with the Lie superalgebra $\mathfrak{g l}_{m \mid n}$. We also use the Sugawara operators to produce algebraically independent generators of the algebra of singular vectors of any generic Verma module at the critical level over the affine Lie superalgebra.
\end{abstract}

School of Mathematics and Statistics

University of Sydney, NSW 2006, Australia

alexm@maths.usyd.edu.au

LAPTH, Chemin de Bellevue, BP 110

F-74941 Annecy-le-Vieux cedex, France

ragoucy@lapp.in2p3.fr 
Dedicated to Grigori Olshanski on his 60th birthday

\section{Introduction}

1.1. MacMahon Master Theorem. A natural quantum analogue of the celebrated MacMahon Master Theorem was proved by Garoufalidis, Lê and Zeilberger in [13]. A few different proofs and generalizations of this analogue have since been found; see Foata and Han [9, 10], Hai and Lorenz [22] and Konvalinka and Pak [28]. In the quantum MacMahon Master Theorem of [13] the numerical matrices are replaced with the right quantum matrices $Z=\left[z_{i j}\right]$ whose matrix elements satisfy some quadratic relations involving a parameter q. As explained in [10], this parameter may be taken to be equal to 1 without a real loss of generality. Then the relations for the matrix elements $z_{i j}$ take the form

$$
\left[z_{i j}, z_{k l}\right]=\left[z_{k j}, z_{i l}\right] \quad \text { for all } i, j, k, l \in\{1, \ldots, N\} \text {, }
$$

where $[x, y]=x y-y x$.

The proof of the quantum MacMahon Master Theorem given in [22] is based on the theory of Koszul algebras. In that approach, (1.1) are the defining relations of the bialgebra end $\mathcal{A}$ associated with the symmetric algebra $\mathcal{A}$ of a vector space. The construction of the bialgebra end $\mathcal{A}$ associated with an arbitrary quadratic algebra or superalgebra $\mathcal{A}$ is due to Manin [30, 31], and the matrices $Z$ satisfying (1.1) are also known as Manin matrices; see [2], [3, [4]. In the super case the construction applied to the symmetric algebra of a $\mathbb{Z}_{2}$-graded vector space (i.e., superspace) of dimension $m+n$ leads to the defining relations of the right quantum superalgebra $\mathcal{M}_{m \mid n}$. This superalgebra is generated by elements $z_{i j}$ with $\mathbb{Z}_{2}$-degree (or parity) $\bar{\imath}+\bar{\jmath}$, where $\bar{\imath}=0$ for $1 \leqslant i \leqslant m$ and $\bar{\imath}=1$ for $m+1 \leqslant i \leqslant m+n$. The defining relations have the form

$$
\left[z_{i j}, z_{k l}\right]=\left[z_{k j}, z_{i l}\right](-1)^{\bar{\imath} \bar{\jmath}+\bar{\imath}+\bar{\jmath} \bar{k}} \quad \text { for all } \quad i, j, k, l \in\{1, \ldots, m+n\},
$$

where $[x, y]=x y-y x(-1)^{\operatorname{deg} x \operatorname{deg} y}$ is the super-commutator of homogeneous elements $x$ and $y$, as presented e.g. in [21, Example 3.14]1. We will call any matrix $Z=\left[z_{i j}\right]$ satisfying (1.2) a Manin matrix.

Our first main result (Theorem 2.2) is an analogue of the MacMahon Master Theorem for the right quantum superalgebra $\mathcal{M}_{m \mid n}$. We will identify the matrix $Z$ with an element of the tensor product superalgebra End $\mathbb{C}^{m \mid n} \otimes \mathcal{M}_{m \mid n}$ by

$$
Z=\sum_{i, j=1}^{m+n} e_{i j} \otimes z_{i j}(-1)^{\bar{\imath} \bar{\jmath}+\bar{\jmath}}
$$

\footnotetext{
${ }^{1}$ The relations given in [21] correspond to our left quantum superalgebra as defined in Sec. 2.2 below.
} 
where the $e_{i j}$ denote the standard matrix units. Taking multiple tensor products

$$
\text { End } \mathbb{C}^{m \mid n} \otimes \ldots \otimes \text { End } \mathbb{C}^{m \mid n} \otimes \mathcal{M}_{m \mid n}
$$

with $k$ copies of End $\mathbb{C}^{m \mid n}$, for any $a=1, \ldots, k$ we will write $Z_{a}$ for the matrix $Z$ corresponding to the $a$-th copy of the endomorphism superalgebra so that the components in all remaining copies are the identity matrices. The symmetric group $\mathfrak{S}_{k}$ acts naturally on the tensor product space $\left(\mathbb{C}^{m \mid n}\right)^{\otimes k}$. We let $H_{k}$ and $A_{k}$ denote the respective images of the normalized symmetrizer and antisymmetrizer

$$
\frac{1}{k !} \sum_{\sigma \in \mathfrak{S}_{k}} \sigma \in \mathbb{C}\left[\mathfrak{S}_{k}\right], \quad \frac{1}{k !} \sum_{\sigma \in \mathfrak{S}_{k}} \operatorname{sgn} \sigma \cdot \sigma \in \mathbb{C}\left[\mathfrak{S}_{k}\right]
$$

in (1.3). Recall that the supertrace of an even matrix $X=\left[x_{i j}\right]$ is defined by

$$
\operatorname{str} X=\sum_{i=1}^{m+n} x_{i i}(-1)^{\bar{\imath}}
$$

Now set

$$
\text { Bos }=1+\sum_{k=1}^{\infty} \operatorname{str} H_{k} Z_{1} \ldots Z_{k}, \quad \text { Ferm }=1+\sum_{k=1}^{\infty}(-1)^{k} \operatorname{str} A_{k} Z_{1} \ldots Z_{k}
$$

where str denotes the supertrace taken with respect to all $k$ copies of End $\mathbb{C}^{m \mid n}$. Our analogue of the MacMahon Master Theorem for the right quantum superalgebra $\mathcal{M}_{m \mid n}$ now reads

$$
\text { Bos } \times \text { Ferm }=1 .
$$

The summands in (1.6) can be written explicitly in terms of the generators $z_{i j}$; see Proposition 2.3 below. In each of the particular cases $n=0$ and $m=0$ the identity (1.7) turns into the quantum MacMahon Master Theorem of [13].

Our proof of the identity (1.7) is based on the use of the matrix form of the defining relations (1.2). These relations can be written as

$$
\left(1-P_{12}\right)\left[Z_{1}, Z_{2}\right]=0
$$

which is considered as an identity in the superalgebra (1.3) with $k=2$ and $P_{12}$ is the image of the transposition $(12) \in \mathfrak{S}_{2}$. The proof of (1.7) is derived from (1.8) by using some elementary properties of the symmetrizers and antisymmetrizers.

1.2. Noncommutative Berezinian. It is well-known that in the super-commutative specialization the expressions (1.6) coincide with the expansions of the Berezinian so that

$$
\text { Ferm }=\operatorname{Ber}(1-Z) \quad \text { and } \quad \operatorname{Bos}=[\operatorname{Ber}(1-Z)]^{-1} ;
$$


see [1], 27], 38]. In order to prove their noncommutative analogues, we introduce the affine right quantum superalgebra $\widehat{\mathcal{M}}_{m \mid n}$. It is generated by a countable set of elements $z_{i j}^{(r)}$ of parity $\bar{\imath}+\bar{\jmath}$, where $r$ runs over the set of positive integers. The defining relations of $\widehat{\mathcal{M}}_{m \mid n}$ take the form

$$
\left(1-P_{12}\right)\left[Z_{1}(u), Z_{2}(u)\right]=0,
$$

where $u$ is a formal variable, and the matrix elements of the matrix $Z(u)=\left[z_{i j}(u)\right]$ are the formal power series

$$
z_{i j}(u)=\delta_{i j}+z_{i j}^{(1)} u+z_{i j}^{(2)} u^{2}+\cdots .
$$

The relation (1.9) is understood in the sense that all coefficients of the powers of $u$ on the left hand side vanish. The formal power series $Z(u)$ in $u$ with matrix coefficients is invertible and we denote by $z_{i j}^{\prime}(u)$ the matrix elements of its inverse so that $Z^{-1}(u)=\left[z_{i j}^{\prime}(u)\right]$. Now we set

$$
\begin{aligned}
\operatorname{Ber} Z(u) & =\sum_{\sigma \in \mathfrak{S}_{m}} \operatorname{sgn} \sigma \cdot z_{\sigma(1) 1}(u) \ldots z_{\sigma(m) m}(u) \\
& \times \sum_{\tau \in \mathfrak{S}_{n}} \operatorname{sgn} \tau \cdot z_{m+1, m+\tau(1)}^{\prime}(u) \ldots z_{m+n, m+\tau(n)}^{\prime}(u) .
\end{aligned}
$$

In the super-commutative specialization Ber $Z(u)$ coincides with the ordinary Berezinian of the matrix $Z(u)$; see [1].

We prove the following expansions of the noncommutative Berezinian, where $Z$ is a Manin matrix:

$$
\operatorname{Ber}(1+u Z)=\sum_{k=0}^{\infty} u^{k} \operatorname{str} A_{k} Z_{1} \ldots Z_{k}
$$

and hence by (1.7)

$$
[\operatorname{Ber}(1-u Z)]^{-1}=\sum_{k=0}^{\infty} u^{k} \operatorname{str} H_{k} Z_{1} \ldots Z_{k} .
$$

Furthermore, we derive the following super-analogues of the Newton identities:

$$
[\operatorname{Ber}(1+u Z)]^{-1} \partial_{u} \operatorname{Ber}(1+u Z)=\sum_{k=0}^{\infty}(-u)^{k} \operatorname{str} Z^{k+1}
$$

which allow one to express the elements $\operatorname{str} Z^{k}$ in terms of the coefficients of either series (1.12) or (1.13). In the even case of $n=0$ the Newton identities were proved in the papers [3. 4] which also contain various generalizations of the matrix algebra properties to the class of Manin matrices. However, the proof of the identities given in these papers relies on the existence of the adjoint matrix and does not immediately extend to the super case. To prove (1.14) we employ instead an appropriate super-extension of the $R$-matrix arguments used in [24]; see also [20]. 
1.3. Segal-Sugawara vectors. The above properties of Manin matrices will be used in our construction of the higher order Sugawara operators for the affine Lie superalgebra $\widehat{\mathfrak{g l}}_{m \mid n}$. The commutation relations of the Lie superalgebra $\widehat{\mathfrak{g l}}_{m \mid n}=\mathfrak{g l}_{m \mid n}\left[t, t^{-1}\right] \oplus \mathbb{C} K$ have the form

$$
\begin{aligned}
{\left[e_{i j}[r], e_{k l}[s]\right]=\delta_{k j} e_{i l}[r+s] } & -\delta_{i l} e_{k j}[r+s](-1)^{(\bar{\imath}+\bar{\jmath})(\bar{k}+\bar{l})} \\
& +K\left(\delta_{k j} \delta_{i l}(-1)^{\bar{\imath}}-\frac{\delta_{i j} \delta_{k l}}{m-n}(-1)^{\bar{\imath}+\bar{k}}\right) r \delta_{r,-s}
\end{aligned}
$$

(assuming $m \neq n$ ), the element $K$ is even and central, and we set $e_{i j}[r]=e_{i j} t^{r}$. To include the case $m=n$ we will re-scale the central element by setting $K=K^{\prime}(n-m)$ so that (1.15) will be written uniformly for all values of $m$ and $n$. We will also consider the extended Lie superalgebra $\widehat{\mathfrak{g l}}_{m \mid n} \oplus \mathbb{C} \tau$, where the element $\tau$ is even and

$$
\left[\tau, e_{i j}[r]\right]=-r e_{i j}[r-1], \quad[\tau, K]=0 .
$$

For any $\kappa \in \mathbb{C}$ the affine vertex algebra $V_{\kappa}\left(\mathfrak{g l}_{m \mid n}\right)$ can be defined as the quotient of the universal enveloping algebra $\mathrm{U}\left(\widehat{\mathfrak{g l}}_{m \mid n}\right)$ by the left ideal generated by $\mathfrak{g l}_{m \mid n}[t]$ and $K-\kappa$; see e.g. [12], [26]. The center of the vertex algebra $V_{\kappa}\left(\mathfrak{g l}_{m \mid n}\right)$ is its subspace spanned by all elements $b \in V_{\kappa}\left(\mathfrak{g l}_{m \mid n}\right)$ such that $\mathfrak{g l}_{m \mid n}[t] b=0$. The axioms of the vertex algebra imply that the center is a commutative associative superalgebra. The center of the vertex algebra $V_{\kappa}\left(\mathfrak{g l}_{m \mid n}\right)$ is trivial for all values of $\kappa$ except for the critical value $\kappa=n-m$ (this corresponds to the evaluation $K^{\prime}=1$ in terms of the re-scaled central element $K^{\prime}$ ). In the latter case the center is big and we denote it by $\mathfrak{z}\left(\widehat{\mathfrak{g l}}_{m \mid n}\right)$. Any element of $\mathfrak{z}\left(\widehat{\mathfrak{g l}}_{m \mid n}\right)$ is called a Segal-Sugawara vector. As a vector superspace, the vertex algebra $V_{n-m}\left(\mathfrak{g l}_{m \mid n}\right)$ can be identified with the universal enveloping algebra $\mathrm{U}\left(t^{-1} \mathfrak{g l}_{m \mid n}\left[t^{-1}\right]\right)$. Moreover, the multiplication in the commutative algebra $\mathfrak{z}\left(\widehat{\mathfrak{g l}}_{m \mid n}\right)$ coincides with the multiplication in $\mathrm{U}\left(t^{-1} \mathfrak{g l}_{m \mid n}\left[t^{-1}\right]\right)$. Therefore, the center $\mathfrak{z}\left(\widehat{\mathfrak{g l}}_{m \mid n}\right)$ is naturally identified with a commutative subalgebra of $\mathrm{U}\left(t^{-1} \mathfrak{g l}_{m \mid n}\left[t^{-1}\right]\right)$.

Another principal result of this paper (Theorem 3.2 and Corollary 3.3) is an explicit construction of several families of Segal-Sugawara vectors. The construction is based on the observation that the matrix elements of the matrix

$$
\tau+\widehat{E}[-1]=\left[\delta_{i j} \tau+e_{i j}[-1](-1)^{\bar{\imath}}\right]
$$

satisfy the defining relations (1.2) of the right quantum superalgebra. That is, $\tau+\widehat{E}[-1]$ is a Manin matrix. We use this fact to show that all coefficients $s_{k l}$ in the expansion

$$
\operatorname{str}(\tau+\widehat{E}[-1])^{k}=s_{k 0} \tau^{k}+s_{k 1} \tau^{k-1}+\cdots+s_{k k}
$$

are Segal-Sugawara vectors. The relations (1.12), (1.13) and (11.14) then lead to explicit formulas for a few other families of Segal-Sugawara vectors. In particular, such vectors 
also occur as the coefficients $b_{k l}$ in the expansion of the Berezinian

$$
\operatorname{Ber}(1+u(\tau+\widehat{E}[-1]))=\sum_{k=0}^{\infty} \sum_{l=0}^{k} b_{k l} u^{k} \tau^{k-l} .
$$

1.4. Commutative subalgebras and higher Gaudin Hamiltonians. Applying the state-field correspondence map to the Segal-Sugawara vectors of any of the families above, we get explicit formulas for elements of the center of the local completion of the universal enveloping algebra $\mathrm{U}\left(\widehat{\mathfrak{g l}}_{m \mid n}\right)$ at the critical level. Such elements are called the (higher order) Sugawara operators. In particular, such operators can be calculated as the Fourier coefficients of the fields $b_{k l}(z)$ defined by the expansion of the normally ordered Berezinian

$$
: \operatorname{Ber}\left(1+u\left(\partial_{z}+\widehat{E}(z)\right)\right):=\sum_{k=0}^{\infty} \sum_{l=0}^{k} b_{k l}(z) u^{k} \partial_{z}^{k-l}
$$

where $\widehat{E}(z)=\left[e_{i j}(z)(-1)^{\bar{\imath}}\right]$ and

$$
e_{i j}(z)=\sum_{r \in \mathbb{Z}} e_{i j}[r] z^{-r-1}
$$

Similarly, all Fourier coefficients of the fields $s_{k l}(z)$ defined by the expansion of the normally ordered supertrace

$$
: \operatorname{str}\left(\partial_{z}+\widehat{E}(z)\right)^{k}:=s_{k 0}(z) \partial_{z}^{k}+s_{k 1}(z) \partial_{z}^{k-1}+\cdots+s_{k k}(z)
$$

are Sugawara operators.

It was observed in [8] that the center of the affine vertex algebra at the critical level is closely related to Hamiltonians of the Gaudin model describing quantum spin chain. The Hamiltonians are obtained by the application of the Sugawara operators to the vacuum vector of the vertex algebra $V_{\kappa}\left(\mathfrak{g l}_{m \mid n}\right)$ with $\kappa=n-m$. Such an application yields elements of the center $\mathfrak{z}\left(\widehat{\mathfrak{g l}}_{m \mid n}\right)$ of $V_{n-m}\left(\mathfrak{g l}_{m \mid n}\right)$. We thus obtain explicit formulas for several families of elements of a commutative subalgebra of $\mathrm{U}\left(t^{-1} \mathfrak{g l}_{m \mid n}\left[t^{-1}\right]\right)$.

Using a duality between the superalgebras $\mathrm{U}\left(t^{-1} \mathfrak{g l}_{m \mid n}\left[t^{-1}\right]\right)$ and $\mathrm{U}\left(\mathfrak{g l}_{m \mid n}[t]\right)$ we also obtain the corresponding families of commuting elements in the superalgebra $\mathrm{U}\left(\mathfrak{g l}_{m \mid n}[t]\right)$. More general families of commutative subalgebras of $\mathrm{U}\left(\mathfrak{g l}_{m \mid n}[t]\right)$ can be brought in by applying some automorphisms of this superalgebra parameterized by diagonal or numerical matrices.

The connection with the Gaudin model is obtained by considering the iterated comultiplication map

$$
\mathrm{U}\left(\mathfrak{g l}_{m \mid n}[t]\right) \rightarrow \mathrm{U}\left(\mathfrak{g l}_{m \mid n}[t]\right) \otimes \ldots \otimes \mathrm{U}\left(\mathfrak{g l}_{m \mid n}[t]\right)
$$


with $k$ copies of $\mathrm{U}\left(\mathfrak{g l}_{m \mid n}[t]\right)$ and then taking the images of elements of a commutative subalgebra of $\mathrm{U}\left(\mathfrak{g l}_{m \mid n}[t]\right)$ in the tensor product of evaluation representations of $\mathrm{U}\left(\mathfrak{g l}_{m \mid n}[t]\right)$. This yields the higher Hamiltonians of the Gaudin model; see Sec. 3.2 below for more details. Such a scheme was used in [39] to give simple and explicit determinant-type formulas for the higher Gaudin Hamiltonians in the case of $\mathfrak{g l}_{n}$; see also [5], 6], 35], 36] and references therein. The results of [5] include a calculation of the eigenvalues of the Sugawara operators in the Wakimoto modules which we believe can be extended to the super case as well with the use of the results of [25].

1.5. Singular vectors in Verma modules. Since the Sugawara operators belong to the center of the local completion of the universal enveloping algebra $\mathrm{U}\left(\widehat{\mathfrak{g l}}_{m \mid n}\right)$ at the critical level, they commute, in particular, with the elements of the Lie superalgebra $\widehat{\mathfrak{g l}}_{m \mid n}$. Therefore, the Sugawara operators form a commuting family of $\widehat{\mathfrak{g l}}_{m \mid n}$-endomorphisms of any Verma module $M(\lambda)$ at the critical level. This means that singular vectors of the Verma module can be constructed by the applications of the Sugawara operators to the highest vector. This approach goes back to the works of Goodman and Wallach [16] and Hayashi [23] where it was applied to the classical Lie algebras and used to prove the KacKazhdan conjecture on the character of the irreducible quotient of $M(\lambda)$.

One of the main results of a recent paper of Gorelik [17, Theorem 1.1] describes the structure of the algebra of singular vectors of generic Verma modules at the critical level over symmetrizable affine Lie superalgebras. Relying on this general theorem we construct two families of algebraically independent generators of the algebra of singular vectors of the generic Verma module $M(\lambda)$ at the critical level over the affine Lie superalgebra $\mathfrak{g l}_{m \mid n}\left[t, t^{-1}\right] \oplus \mathbb{C} K \oplus \mathbb{C} d$ (Theorem 3.10 and Corollary 3.11).

Since the first version of this paper appeared in the arXiv in 2009, some related results concerning the center of the affine vertex algebra at the critical level (the Feigin-Frenkel center) associated with the simple Lie algebras of types $B, C$ and $D$ have been obtained. In particular, explicit generators of the center were constructed in [33] and their HarishChandra images (the eigenvalues in Wakimoto modules) were calculated in [34].

We are grateful to Lucy Gow for help with calculations in the case $m=n$. E. R. was at the University of Sydney during the completion of this work, he wishes to warmly thank the School of Mathematics and Statistics for hospitality. We acknowledge the financial support of the Australian Research Council. 


\section{Manin matrices over superalgebras}

\subsection{MacMahon Master Theorem}

As defined in the Introduction, the right quantum superalgebra $\mathcal{M}_{m \mid n}$ is generated by the elements $z_{i j}$ with $1 \leqslant i, j \leqslant m+n$ such that the parity of $z_{i j}$ is $\bar{\imath}+\bar{\jmath}$. The defining relations are given in (1.2). In what follows we will use the matrix form (1.8) of these relations. In order to explain this notation in more detail, consider the superalgebra

$$
\text { End } \mathbb{C}^{m \mid n} \otimes \ldots \otimes \text { End } \mathbb{C}^{m \mid n} \otimes \mathcal{M}_{m \mid n}
$$

with $k$ copies of End $\mathbb{C}^{m \mid n}$. For each $a \in\{1, \ldots, k\}$ the element $Z_{a}$ of the superalgebra (2.1) is defined by the formula

$$
Z_{a}=\sum_{i, j=1}^{m+n} 1^{\otimes(a-1)} \otimes e_{i j} \otimes 1^{\otimes(k-a)} \otimes z_{i j}(-1)^{\bar{i} \bar{\jmath}+\bar{\jmath}} .
$$

Using the natural action of $\mathfrak{S}_{k}$ on $\left(\mathbb{C}^{m \mid n}\right)^{\otimes k}$ we represent any permutation $\sigma \in \mathfrak{S}_{k}$ as an element $P_{\sigma}$ of the superalgebra (2.1) with the identity component in $\mathcal{M}_{m \mid n}$. In particular, the transposition $(a b)$ with $a<b$ corresponds to the element

$$
P_{a b}=\sum_{i, j=1}^{m+n} 1^{\otimes(a-1)} \otimes e_{i j} \otimes 1^{\otimes(b-a-1)} \otimes e_{j i} \otimes 1^{\otimes(k-b)} \otimes 1(-1)^{\bar{\jmath}},
$$

which allows one to determine $P_{\sigma}$ by writing an arbitrary $\sigma \in \mathfrak{S}_{k}$ as a product of transpositions. Recall also that if $x, x^{\prime}$ are homogeneous elements of a superalgebra $\mathcal{A}$ and $y, y^{\prime}$ are homogeneous elements of a superalgebra $\mathcal{B}$ then the product in the superalgebra $\mathcal{A} \otimes \mathcal{B}$ is calculated with the use of the sign rule

$$
(x \otimes y)\left(x^{\prime} \otimes y^{\prime}\right)=\left(x x^{\prime} \otimes y y^{\prime}\right)(-1)^{\operatorname{deg} y \operatorname{deg} x^{\prime}} .
$$

Note that all the elements $Z_{a}$ and $P_{a b}$ are even and $P_{a b}$ commutes with $Z_{c}$ if $c \neq a, b$. Moreover, $P_{a b} Z_{a}=Z_{b} P_{a b}$.

For each $a=1, \ldots, k$ the supertrace $\operatorname{str}_{a}$ with respect to the $a$-th copy of End $\mathbb{C}^{m \mid n}$ in (2.1) is the linear map

$$
\operatorname{str}_{a}:\left(\text { End } \mathbb{C}^{m \mid n}\right)^{\otimes k} \otimes \mathcal{M}_{m \mid n} \rightarrow\left(\text { End } \mathbb{C}^{m \mid n}\right)^{\otimes k-1} \otimes \mathcal{M}_{m \mid n},
$$

defined by

$$
\begin{aligned}
\operatorname{str}_{a}: x_{1} \otimes \ldots \otimes x_{a-1} & \otimes e_{i j} \otimes x_{a+1} \otimes \ldots \otimes x_{k} \otimes y \\
& \mapsto \delta_{i j} x_{1} \otimes \ldots \otimes x_{a-1} \otimes x_{a+1} \otimes \ldots \otimes x_{k} \otimes y(-1)^{\bar{\imath}} .
\end{aligned}
$$


In the case $k=1$ this definition clearly agrees with (1.5). The subscripts like $\operatorname{str}_{1, \ldots, k}$ of the supertrace will indicate that it is taken over the copies $1,2, \ldots, k$ of the superalgebra End $\mathbb{C}^{m \mid n}$ in (2.1). The following cyclic property of the supertrace will often be used: if $X=\left[x_{i j}\right]$ and $Y=\left[y_{i j}\right]$ are even matrices with pairwise super-commuting entries, $\left[x_{i j}, y_{k l}\right]=0$, then

$$
\operatorname{str}(X Y-Y X)=0
$$

We will need the properties of Manin matrices given in the next proposition. In the even case $(n=0)$ they are formulated in [4, Proposition 18]. Recall that $H_{k}$ and $A_{k}$ denote the respective images of the symmetrizer and antisymmetrizer in (2.1); see (1.4).

Proposition 2.1. We have the identities in the superalgebra (2.1),

$$
A_{k} Z_{1} \ldots Z_{k} A_{k}=A_{k} Z_{1} \ldots Z_{k}
$$

and

$$
H_{k} Z_{1} \ldots Z_{k} H_{k}=Z_{1} \ldots Z_{k} H_{k}
$$

Proof. To prove (2.3) it suffices to show that for any $a=1, \ldots, k-1$ we have

$$
A_{k} Z_{1} \ldots Z_{k} P_{a a+1}=-A_{k} Z_{1} \ldots Z_{k}
$$

Since $A_{k}=A_{k}\left(1-P_{a a+1}\right) / 2$, it is enough to consider the case $k=2$. The relation (2.5) then reads

$$
\frac{1-P_{12}}{2} Z_{1} Z_{2} P_{12}=-\frac{1-P_{12}}{2} Z_{1} Z_{2}
$$

which an equivalent form of (1.8). Similarly, the proof of (2.4) reduces to checking that

$$
P_{a a+1} Z_{1} \ldots Z_{k} H_{k}=Z_{1} \ldots Z_{k} H_{k}
$$

This follows again from (1.8) written in the form

$$
P_{12} Z_{1} Z_{2} \frac{1+P_{12}}{2}=Z_{1} Z_{2} \frac{1+P_{12}}{2}
$$

We are now in a position to prove the MacMahon Master Theorem for the right quantum superalgebra $\mathcal{M}_{m \mid n}$. We use the notation (1.6).

Theorem 2.2. We have the identity

Bos $\times$ Ferm $=1$. 
Proof. It is sufficient to show that for any integer $k \geqslant 1$ we have the identity in the superalgebra (2.1)

$$
\sum_{r=0}^{k}(-1)^{k-r} \operatorname{str}_{1, \ldots, r} H_{r} Z_{1} \ldots Z_{r} \times \operatorname{str}_{r+1, \ldots, k} A_{\{r+1, \ldots, k\}} Z_{r+1} \ldots Z_{k}=0
$$

where $A_{\{r+1, \ldots, k\}}$ denotes the antisymmetrizer in (2.1) over the copies of End $\mathbb{C}^{m \mid n}$ labeled by $r+1, \ldots, k$. The identity can be written as

$$
\sum_{r=0}^{k}(-1)^{r} \operatorname{str}_{1, \ldots, k} H_{r} A_{\{r+1, \ldots, k\}} Z_{1} \ldots Z_{k}=0
$$

Our next step is to show that the product of the symmetrizer and antisymmetrizer in (2.6) can be replaced as follows:

$$
H_{r} A_{\{r+1, \ldots, k\}} \mapsto \frac{r(k-r+1)}{k} H_{r} A_{\{r, \ldots, k\}}+\frac{(r+1)(k-r)}{k} H_{r+1} A_{\{r+1, \ldots, k\}} .
$$

Indeed, the right hand side of (2.7) equals

$$
\begin{aligned}
& \frac{r(k-r+1)}{k} H_{r}\left(\frac{1}{k-r+1} A_{\{r+1, \ldots, k\}}-\frac{k-r}{k-r+1} A_{\{r+1, \ldots, k\}} P_{r, r+1} A_{\{r+1, \ldots, k\}}\right) \\
+ & \frac{(r+1)(k-r)}{k}\left(\frac{1}{r+1} H_{r}+\frac{r}{r+1} H_{r} P_{r, r+1} H_{r}\right) A_{\{r+1, \ldots, k\}} .
\end{aligned}
$$

Since $H_{r}$ commutes with $A_{\{r+1, \ldots, k\}}$, using the cyclic property of the supertrace we get

$$
\begin{aligned}
\operatorname{str}_{1, \ldots, k} H_{r} A_{\{r+1, \ldots, k\}} P_{r, r+1} A_{\{r+1, \ldots, k\}} Z_{1} & \ldots Z_{k} \\
& =\operatorname{str}_{1, \ldots, k} H_{r} P_{r, r+1} A_{\{r+1, \ldots, k\}} Z_{1} \ldots Z_{k} A_{\{r+1, \ldots, k\}} .
\end{aligned}
$$

Now apply the first relation of Proposition 2.1 to write this element as

$$
\operatorname{str}_{1, \ldots, k} H_{r} P_{r, r+1} A_{\{r+1, \ldots, k\}} Z_{1} \ldots Z_{k}
$$

Similarly, using the second relation of Proposition 2.1 and the cyclic property of the supertrace, we get

$$
\operatorname{str}_{1, \ldots, k} H_{r} P_{r, r+1} H_{r} A_{\{r+1, \ldots, k\}} Z_{1} \ldots Z_{k}=\operatorname{str}_{1, \ldots, k} H_{r} P_{r, r+1} A_{\{r+1, \ldots, k\}} Z_{1} \ldots Z_{k}
$$

thus showing that the left hand side of (2.6) remains unchanged after the replacement (2.7). Then the telescoping sum in (2.6) vanishes and the proof is complete. 
We now give explicit formulas for the summands in (1.6) in terms of the generators $z_{i j}$ of the superalgebra $\mathcal{M}_{m \mid n}$. We fix some notation first. Given any permutation $\sigma \in \mathfrak{S}_{k}$ consider the product of the elements $P_{\sigma}$ and $e_{i_{1} j_{1}} \otimes \ldots \otimes e_{i_{k} j_{k}} \otimes 1$ of the superalgebra (2.1). We define the coefficient $\varphi(\sigma, I, J)$ by writing this product in the form

$$
P_{\sigma}\left(e_{i_{1} j_{1}} \otimes \ldots \otimes e_{i_{k} j_{k}} \otimes 1\right)=\varphi(\sigma, I, J) \cdot e_{i_{\sigma^{-1}(1)} j_{1}} \otimes \ldots \otimes e_{i_{\sigma^{-1}(k)} j_{k}} \otimes 1
$$

where $I=\left\{i_{1}, \ldots, i_{k}\right\}$ and $J=\left\{j_{1}, \ldots, j_{k}\right\}$ so that $\varphi(\sigma, I, J)$ equals 1 or -1 depending on the multisets $I, J$ and the permutation $\sigma$. Similarly, define the $\operatorname{sign} \psi(\sigma, I, J)$ by the relation

$$
\left(e_{i_{1} j_{1}} \otimes \ldots \otimes e_{i_{k} j_{k}} \otimes 1\right) P_{\sigma^{-1}}=\psi(\sigma, I, J) \cdot e_{i_{1} j_{\sigma^{-1}}(1)} \otimes \ldots \otimes e_{i_{k} j_{\sigma^{-1}}(k)} \otimes 1
$$

We also set

$$
\gamma(I, J)=\sum_{a} \bar{\imath}_{a} \bar{\jmath}_{a}+\sum_{a<b}\left(\bar{\imath}_{a}+\bar{\jmath}_{a}\right)\left(\bar{\imath}_{b}+\bar{\jmath}_{b}\right)
$$

and let $\alpha_{i}$ denote the multiplicity of index $i$ in the given multiset $I$.

Proposition 2.3. We have the relation

$$
\begin{aligned}
\operatorname{str}_{1, \ldots, k} A_{k} Z_{1} \ldots Z_{k} & =\sum_{I} \frac{1}{\alpha_{m+1} ! \ldots \alpha_{m+n} !} \\
& \times \sum_{\sigma \in \mathfrak{S}_{k}} \operatorname{sgn} \sigma \cdot \varphi(\sigma, \sigma I, I) \cdot z_{i_{\sigma(1)} i_{1}} \ldots z_{i_{\sigma(k)} i_{k}}(-1)^{\gamma(\sigma I, I)},
\end{aligned}
$$

summed over multisets $I=\left\{i_{1} \geqslant \cdots \geqslant i_{l} \geqslant m+1>i_{l+1}>\cdots>i_{k}\right\}$ with $l=0, \ldots, k$, where $\sigma I=\left\{i_{\sigma(1)}, \ldots, i_{\sigma(k)}\right\}$. Moreover,

$$
\begin{aligned}
\operatorname{str}_{1, \ldots, k} Z_{1} \ldots Z_{k} H_{k} & =\sum_{I} \frac{1}{\alpha_{1} ! \ldots \alpha_{m} !} \\
& \times \sum_{\sigma \in \mathfrak{S}_{k}} \psi(\sigma, I, \sigma I) \cdot z_{i_{1} i_{\sigma(1)}} \ldots z_{i_{k} i_{\sigma(k)}}(-1)^{\gamma(I, \sigma I)},
\end{aligned}
$$

summed over multisets $I=\left\{i_{1} \leqslant \cdots \leqslant i_{l} \leqslant m<i_{l+1}<\cdots<i_{k}\right\}$ with $l=0, \ldots, k$.

Proof. Write

$$
A_{k} Z_{1} \ldots Z_{k}=\sum_{I, J} e_{i_{1} j_{1}} \otimes \ldots \otimes e_{i_{k} j_{k}} \otimes Z_{j_{1} \ldots j_{k}}^{i_{1} \ldots i_{k}}
$$

summed over multisets $I=\left\{i_{1}, \ldots, i_{k}\right\}$ and $J=\left\{j_{1}, \ldots, j_{k}\right\}$, where $Z_{j_{1} \ldots j_{k}}^{i_{1} \ldots i_{k}} \in \mathcal{M}_{m \mid n}$. By Proposition 2.1, for each $a=1, \ldots, k-1$ we have

$$
P_{a, a+1} A_{k} Z_{1} \ldots Z_{k}=A_{k} Z_{1} \ldots Z_{k} P_{a, a+1}=-A_{k} Z_{1} \ldots Z_{k}
$$


Therefore, the coefficients in the expansion (2.10) satisfy

$$
Z_{j_{1} \ldots j_{k}}^{i_{1} \ldots i_{a+1} i_{a} \ldots i_{k}}=-Z_{j_{1} \ldots j_{k}}^{i_{1} \ldots i_{k}}(-1)^{\bar{\imath}_{a} \bar{\imath}_{a+1}+\bar{\imath}_{a} \bar{\jmath}_{a}+\bar{\imath}_{a+1} \bar{\jmath}_{a}}
$$

and

$$
Z_{j_{1} \ldots j_{a+1} j_{a} \ldots j_{k}}^{i_{1} \ldots i_{k}}=-Z_{j_{1} \ldots j_{k}}^{i_{1} \ldots i_{k}}(-1)^{\bar{\jmath}_{a} \bar{\jmath}_{a+1}+\bar{\jmath}_{a} \bar{a}_{a+1}+\bar{\jmath}_{a+1} \bar{c}_{a+1}} .
$$

Either of these relations implies that if $i_{a}=i_{a+1}$ then

$$
Z_{i_{1} \ldots i_{a} i_{a+1} \ldots i_{k}}^{i_{1} \ldots i_{a} i_{a+1} \ldots i_{k}}=-Z_{i_{1} \ldots i_{a} i_{a+1} \ldots i_{k}}^{i_{1} \ldots i_{i} i_{a+1} \ldots i_{k}}(-1)^{\bar{\imath}_{a}}
$$

so that the coefficient vanishes if $\bar{\imath}_{a}=0$. Moreover, for any multiset $I$ we have

$$
Z_{i_{1} \ldots i_{a+1} i_{a} \ldots i_{k}}^{i_{1} \ldots i_{a+1} i_{a} \ldots i_{k}}=Z_{i_{1} \ldots i_{k}}^{i_{1} \ldots i_{k}}
$$

By the definition of the supertrace, we have

$$
\operatorname{str}_{1, \ldots, k} A_{k} Z_{1} \ldots Z_{k}=\sum_{I} Z_{i_{1} \ldots i_{k}}^{i_{1} \ldots i_{k}}(-1)^{\bar{\imath}_{1}+\cdots+\bar{\imath}_{k}}
$$

Using (2.11) and (2.12), we can rewrite this expression by taking the summation over multisets of the form $I=\left\{i_{1} \geqslant \cdots \geqslant i_{l} \geqslant m+1>i_{l+1}>\cdots>i_{k}\right\}$ with $l=0, \ldots, k$ so that

$$
\operatorname{str}_{1, \ldots, k} A_{k} Z_{1} \ldots Z_{k}=\sum_{I} \frac{k !}{\alpha_{m+1} ! \ldots \alpha_{m+n} !} Z_{i_{1} \ldots i_{k}}^{i_{1} \ldots i_{k}}(-1)^{\bar{\imath}_{1}+\cdots+\bar{\imath}_{k}} .
$$

On the other hand, multiplying the elements of the superalgebra (2.1) we find that

$$
\begin{aligned}
A_{k} Z_{1} \ldots Z_{k}= & \frac{1}{k !} \sum_{\sigma \in \mathfrak{S}_{k}} \operatorname{sgn} \sigma \cdot P_{\sigma} \\
& \times \sum_{I, J} e_{i_{1} j_{1}} \otimes \ldots \otimes e_{i_{k} j_{k}} \otimes z_{i_{1} j_{1}} \ldots z_{i_{k} j_{k}}(-1)^{\sum_{a}\left(\bar{\imath}_{a} \bar{\jmath}_{a}+\bar{\jmath}_{a}\right)+\sum_{a<b}\left(\bar{\imath}_{a}+\bar{\jmath}_{a}\right)\left(\bar{\imath}_{b}+\bar{\jmath}_{b}\right)} .
\end{aligned}
$$

Then using the notation (2.8) and (2.9) we can write

$$
Z_{j_{1} \ldots j_{k}}^{i_{1} \ldots i_{k}}=\frac{1}{k !} \sum_{\sigma \in \mathfrak{S}_{k}} \operatorname{sgn} \sigma \cdot \varphi(\sigma, \sigma I, J) \cdot z_{i_{\sigma(1)} j_{1}} \ldots z_{i_{\sigma(k)} j_{k}}(-1)^{\gamma(\sigma I, J)}(-1)^{\bar{j}_{1}+\cdots+\bar{\jmath}_{k}}
$$

which completes the proof of the first relation. The second relation is verified by a similar argument with the use of the identities

$$
P_{a, a+1} Z_{1} \ldots Z_{k} H_{k}=Z_{1} \ldots Z_{k} H_{k} P_{a, a+1}=Z_{1} \ldots Z_{k} H_{k}
$$

implied by Proposition 2.1 , 
Remark 2.4. (i) Relation (2.12) can be used to get alternative formulas for the elements $\operatorname{str}_{1, \ldots, k} A_{k} Z_{1} \ldots Z_{k}$ and $\operatorname{str}_{1, \ldots, k} Z_{1} \ldots Z_{k} H_{k}$ with the summation over multisets of the form $\left\{i_{1}<\cdots<i_{l}<m+1 \leqslant i_{l+1} \leqslant \cdots \leqslant i_{k}\right\}$ and $\left\{i_{1}>\cdots>i_{l}>m \geqslant i_{l+1} \geqslant \cdots \geqslant i_{k}\right\}$, respectively.

(ii) Taking the particular cases $n=0$ and $m=0$ in Theorem 2.2 we thus get a new proof of the MacMahon Master Theorem for the right quantum algebra; cf. [9], [13], [28]. Some other noncommutative versions of the theorem associated with quantum algebras or classical Lie algebras can be found e.g. in [20] and [32, Ch. 7]; see also references therein.

\subsection{Affine right quantum superalgebras}

In accordance to the definition we gave in the Introduction, the affine right quantum superalgebra $\widehat{\mathcal{M}}_{m \mid n}$ is generated by the elements $z_{i j}^{(r)}$ of parity $\bar{\imath}+\bar{\jmath}$, where $r$ runs over the set of positive integers and $1 \leqslant i, j \leqslant m+n$. The defining relations (1.9) can be written more explicitly as follows: for all positive integers $p$ we have

$$
\sum_{r+s=p}\left(\left[z_{i j}^{(r)}, z_{k l}^{(s)}\right]-\left[z_{k j}^{(r)}, z_{i l}^{(s)}\right](-1)^{\bar{\imath} \bar{\jmath}+\bar{k}+\bar{\jmath} \bar{k}}\right)=0,
$$

summed over nonnegative integers $r$ and $s$, where we set $z_{i j}^{(0)}=\delta_{i j}$. Equivalently, in terms of the formal power series (1.10) they take the form

$$
\left[z_{i j}(u), z_{k l}(u)\right]=\left[z_{k j}(u), z_{i l}(u)\right](-1)^{\overline{\bar{\jmath}}+\bar{\imath} \bar{k}+\bar{\jmath} \bar{k}}, \quad i, j, k, l \in\{1, \ldots, m+n\} .
$$

We will keep the notation $z_{i j}^{\prime}(u)$ for the entries of the inverse matrix $Z^{-1}(u)$.

Proposition 2.5. The mapping

$$
z_{i j}(u) \mapsto \delta_{i j}+z_{i j} u
$$

defines a surjective homomorphism $\widehat{\mathcal{M}}_{m \mid n} \rightarrow \mathcal{M}_{m \mid n}$. Moreover, the mapping

$$
z_{i j} \mapsto z_{i j}^{(1)}
$$

defines an embedding $\mathcal{M}_{m \mid n} \hookrightarrow \widehat{\mathcal{M}}_{m \mid n}$.

Proof. This follows easily from the defining relations of $\mathcal{M}_{m \mid n}$ and $\widehat{\mathcal{M}}_{m \mid n}$. The injectivity of the map (2.16) follows from the observation that the composition of (2.16) and (2.15) yields the identity map on $\mathcal{M}_{m \mid n}$.

We will identify $\mathcal{M}_{m \mid n}$ with a subalgebra of $\widehat{\mathcal{M}}_{m \mid n}$ via the embedding (2.16). 
Definition 2.6. The Berezinian of the matrix $Z(u)$ is a formal power series in $u$ with coefficients in $\widehat{\mathcal{M}}_{m \mid n}$ defined by the formula

$$
\begin{aligned}
\operatorname{Ber} Z(u) & =\sum_{\sigma \in \mathfrak{S}_{m}} \operatorname{sgn} \sigma \cdot z_{\sigma(1) 1}(u) \ldots z_{\sigma(m) m}(u) \\
& \times \sum_{\tau \in \mathfrak{S}_{n}} \operatorname{sgn} \tau \cdot z_{m+1, m+\tau(1)}^{\prime}(u) \ldots z_{m+n, m+\tau(n)}^{\prime}(u) .
\end{aligned}
$$

The image of the Berezinian Ber $Z(u)$ under the homomorphism (2.15) will be denoted by $\operatorname{Ber}(1+u Z)$. This is a formal power series in $u$ with coefficients in the right quantum superalgebra $\mathcal{M}_{m \mid n}$.

An alternative formula for the Berezinian Ber $Z(u)$ will be given in Corollary 2.16 below.

Our goal now is to derive a quasideterminant factorization of $\operatorname{Ber} Z(u)$ and then use it in the proof of the identities (1.12)-(1.14). We start by providing some symmetries of the superalgebra $\widehat{\mathcal{M}}_{m \mid n}$.

Lemma 2.7. If $Z$ is a Manin matrix, then for any positive integer $r$ the following identity holds:

$$
\left(1-P_{12}\right) \sum_{k+l=r}\left[Z_{1}^{k}, Z_{2}^{l}\right]=0
$$

where $k$ and $l$ run over nonnegative integers.

Proof. We use induction on $r$. The identity is trivial for $r=1$, while for $r=2$ it is equivalent to the definition of a Manin matrix; see (1.8). Suppose that $r \geqslant 3$. Note first that using the induction hypothesis we get

$$
\begin{aligned}
& \left(1-P_{12}\right) Z_{2} Z_{1} \sum_{k+l=r-2}\left[Z_{1}^{k}, Z_{2}^{l}\right]=\left(1-P_{12}\right) Z_{1} Z_{2} \sum_{k+l=r-2}\left[Z_{1}^{k}, Z_{2}^{l}\right] \\
= & \left(1-P_{12}\right) Z_{1} Z_{2} P_{12} \sum_{k+l=r-2}\left[Z_{1}^{k}, Z_{2}^{l}\right]=-\left(1-P_{12}\right) Z_{2} Z_{1} \sum_{k+l=r-2}\left[Z_{1}^{k}, Z_{2}^{l}\right]
\end{aligned}
$$

and so

$$
\left(1-P_{12}\right) Z_{2} Z_{1} \sum_{k+l=r}\left[Z_{1}^{k-1}, Z_{2}^{l-1}\right]=0
$$

Now for $k \geqslant 1$ write

$$
\left[Z_{1}^{k}, Z_{2}^{l}\right]=\left[Z_{1}, Z_{2}^{l}\right] Z_{1}^{k-1}+Z_{1}\left[Z_{1}^{k-1}, Z_{2}^{l}\right]
$$

If $l \geqslant 1$ then we also have

$$
\left[Z_{1}, Z_{2}^{l}\right] Z_{1}^{k-1}=\left[Z_{1}, Z_{2}\right] Z_{2}^{l-1} Z_{1}^{k-1}+Z_{2}\left[Z_{1}, Z_{2}^{l-1}\right] Z_{1}^{k-1}
$$


Therefore, due to (1.8) we obtain

$$
\left(1-P_{12}\right) \sum_{k+l=r}\left[Z_{1}^{k}, Z_{2}^{l}\right]=\left(1-P_{12}\right) \sum_{k+l=r}\left(Z_{2}\left[Z_{1}, Z_{2}^{l-1}\right] Z_{1}^{k-1}+Z_{1}\left[Z_{1}^{k-1}, Z_{2}^{l}\right]\right),
$$

where $k$ and $l$ run over positive integers. By the induction hypothesis,

$$
\begin{aligned}
\left(1-P_{12}\right) \sum_{k+l=r} Z_{1}\left[Z_{1}^{k-1}, Z_{2}^{l}\right] & =\left(1-P_{12}\right) Z_{1} P_{12} \sum_{k+l=r}\left[Z_{1}^{k-1}, Z_{2}^{l}\right] \\
& =-\left(1-P_{12}\right) \sum_{k+l=r} Z_{2}\left[Z_{1}^{k-1}, Z_{2}^{l}\right] .
\end{aligned}
$$

Now for $k \geqslant 2$ write

$$
\left[Z_{1}^{k-1}, Z_{2}^{l}\right]=\left[Z_{1}, Z_{2}^{l}\right] Z_{1}^{k-2}+Z_{1}\left[Z_{1}^{k-2}, Z_{2}^{l}\right] .
$$

Hence, taking appropriate restrictions on the summation indices $k$ and $l$ we get

$$
\begin{aligned}
& \left(1-P_{12}\right) \sum_{k+l=r}\left[Z_{1}^{k}, Z_{2}^{l}\right] \\
= & \left(1-P_{12}\right) \sum_{k+l=r}\left(Z_{2}\left[Z_{1}, Z_{2}^{l-1}\right] Z_{1}^{k-1}-Z_{2}\left[Z_{1}, Z_{2}^{l}\right] Z_{1}^{k-2}-Z_{2} Z_{1}\left[Z_{1}^{k-2}, Z_{2}^{l}\right]\right)
\end{aligned}
$$

which is zero by (2.19).

Proposition 2.8. The mapping

$$
\omega: Z(u) \mapsto Z^{-1}(-u)
$$

defines an involutive automorphism of the superalgebra $\widehat{\mathcal{M}}_{m \mid n}$ which is identical on the subalgebra $\mathcal{M}_{m \mid n}$.

Proof. Set $\check{Z}=1-Z(-u)$ so that $\check{Z}$ is a formal power series in $u$ without a constant term. Then $Z^{-1}(-u)$ can be written in the form

$$
Z^{-1}(-u)=\sum_{k=0}^{\infty} \check{Z}^{k} .
$$

Note that the right hand side is a well-defined power series in $u$ with coefficients in $\widehat{\mathcal{M}}_{m \mid n}$. By Lemma 2.7, for any positive integer $r$ we have

$$
\left(1-P_{12}\right) \sum_{k+l=r}\left[\check{Z}_{1}^{k}, \check{Z}_{2}^{l}\right]=0,
$$

which implies

$$
\left(1-P_{12}\right)\left[Z_{1}^{-1}(-u), Z_{2}^{-1}(-u)\right]=0
$$

thus proving that $\omega$ is a homomorphism.

Applying $\omega$ to both sides of $\omega(Z(u)) Z(-u)=1$ we get $\omega^{2}(Z(u)) Z^{-1}(u)=1$, so that $\omega$ is an involutive automorphism of $\widehat{\mathcal{M}}_{m \mid n}$. The second statement is clear. 
We also introduce the affine left quantum superalgebra $\widehat{\mathcal{M}}_{m \mid n}^{\circ}$. It is generated by a countable set of elements $y_{i j}^{(r)}$ of parity $\bar{\imath}+\bar{\jmath}$, where $r$ runs over the set of positive integers. The defining relations of $\widehat{\mathcal{M}}_{m \mid n}^{\circ}$ take the form

$$
\left[Y_{1}(u), Y_{2}(u)\right]\left(1-P_{12}\right)=0
$$

where $u$ is a formal variable, and the matrix elements of the matrix $Y(u)=\left[y_{i j}(u)\right]$ are the formal power series

$$
y_{i j}(u)=\delta_{i j}+y_{i j}^{(1)} u+y_{i j}^{(2)} u^{2}+\cdots .
$$

The defining relations (2.21) can be written as

$$
\left[y_{i j}(u), y_{k l}(u)\right]+\left[y_{k j}(u), y_{i l}(u)\right](-1)^{\bar{\imath} \bar{\jmath}+\bar{\imath} \bar{k}+\bar{\jmath} \bar{k}}=0, \quad i, j, k, l \in\{1, \ldots, m+n\},
$$

or, equivalently, for all positive integers $p$ we have

$$
\sum_{r+s=p}\left(\left[y_{i j}^{(r)}, y_{k l}^{(s)}\right]+\left[y_{k j}^{(r)}, y_{i l}^{(s)}\right](-1)^{\bar{\imath} \bar{\jmath}+\bar{\imath} \bar{k}+\bar{\jmath} \bar{k}}\right)=0
$$

summed over nonnegative integers $r$ and $s$, where we set $y_{i j}^{(0)}=\delta_{i j}$.

It is straightforward to verify that the superalgebra $\widehat{\mathcal{M}}_{m \mid n}^{\circ}$ is isomorphic to the affine right quantum superalgebra $\widehat{\mathcal{M}}_{m \mid n}$. An isomorphism can be given by the supertransposition map $Y(u) \mapsto Z(u)^{t}$ so that $y_{i j}(u) \mapsto z_{j i}(u)(-1)^{\bar{\imath} \bar{\jmath}+\bar{\imath}}$.

Proposition 2.9. The mapping

$$
\zeta: y_{i j}(u) \mapsto z_{m+n-i+1, m+n-j+1}^{\prime}(u)
$$

defines an isomorphism $\widehat{\mathcal{M}}_{n \mid m}^{\circ} \rightarrow \widehat{\mathcal{M}}_{m \mid n}$.

Proof. Observe that the mapping $y_{i j}(u) \mapsto z_{m+n-i+1, m+n-j+1}(-u)$ defines an isomorphism $\widehat{\mathcal{M}}_{n \mid m}^{\circ} \rightarrow \widehat{\mathcal{M}}_{m \mid n}$. This follows easily from the defining relations of the affine left and right quantum superalgebras. It remains to note that $\zeta$ is the composition of this isomorphism and the automorphism $\omega$ defined in Proposition 2.8.

We will now adapt the arguments used by Gow [18, 19] for the Yangian of the Lie superalgebra $\mathfrak{g l}_{m \mid n}$ to derive a quasideterminant decomposition of the Berezinian $\operatorname{Ber} Z(u)$; see Definition 2.6.

If $A=\left[a_{i j}\right]$ is a square matrix over a ring with 1 , then its $i j$-th quasideterminant is defined if $A$ is invertible and the $j i$-th entry $\left(A^{-1}\right)_{j i}$ is an invertible element of the ring. The $i j$-th quasideterminant is then given by

$$
\left|\begin{array}{lllll}
a_{11} & \ldots & a_{1 j} & \ldots & a_{1 N} \\
& \ldots & & \ldots & \\
a_{i 1} & \ldots & a_{i j} & \ldots & a_{i N} \\
& \ldots & & \ldots & \\
a_{N 1} & \ldots & a_{N j} & \ldots & a_{N N}
\end{array}\right|=\left(\left(A^{-1}\right)_{j i}\right)^{-1} .
$$


We refer the reader to [14, [15] and references therein for the properties and applications of the quasideterminants.

We will need the Gauss decompositions of the matrices $Z(u)$ and $Y(u)$. There exist unique matrices $D(u), E(u)$ and $F(u)$ whose entries are formal power series in $u$ with coefficients in $\widehat{\mathcal{M}}_{m \mid n}$ such that $D(u)=\operatorname{diag}\left[d_{1}(u), \ldots, d_{m+n}(u)\right]$ and

$$
E(u)=\left[\begin{array}{cccc}
1 & e_{12}(u) & \ldots & e_{1, m+n}(u) \\
0 & 1 & \ldots & e_{2, m+n}(u) \\
\vdots & \vdots & \ddots & \vdots \\
0 & 0 & \ldots & 1
\end{array}\right], \quad F(u)=\left[\begin{array}{cccc}
1 & 0 & \ldots & 0 \\
f_{21}(u) & 1 & \ldots & 0 \\
\vdots & \vdots & \ddots & \vdots \\
f_{m+n, 1}(u) & f_{m+n, 2}(u) & \ldots & 1
\end{array}\right]
$$

satisfying $Z(u)=F(u) D(u) E(u)$. Explicit formulas for the entries of the matrices $D(u)$, $E(u)$ and $F(u)$ can be given in terms of quasideterminants; see [14]. In particular,

$$
d_{i}(u)=\left|\begin{array}{cccc}
z_{11}(u) & \ldots & z_{1, i-1}(u) & z_{1 i}(u) \\
\vdots & \ddots & \vdots & \vdots \\
z_{i-1,1}(u) & \ldots & z_{i-1, i-1}(u) & z_{i-1, i}(u) \\
z_{i 1}(u) & \ldots & z_{i, i-1}(u) & z_{i i}(u)
\end{array}\right|
$$

for $i=1, \ldots, m+n$.

We write the Gauss decomposition of the matrix $Y(u)$ as $Y(u)=F^{\circ}(u) D^{\circ}(u) E^{\circ}(u)$ so that the corresponding entries $d_{i}^{\circ}(u), e_{i j}^{\circ}(u), f_{i j}^{\circ}(u)$ of the matrices $D^{\circ}(u), E^{\circ}(u)$ and $F^{\circ}(u)$ are formal power series in $u$ with coefficients in $\widehat{\mathcal{M}}_{m \mid n}^{\circ}$. These entries are found by the same formulas as above with the $z_{i j}(u)$ respectively replaced by $y_{i j}(u)$.

Lemma 2.10. Under the isomorphism $\zeta: \widehat{\mathcal{M}}_{n \mid m}^{\circ} \rightarrow \widehat{\mathcal{M}}_{m \mid n}$ defined in (2.25) we have

$$
\zeta: d_{k}^{\circ}(u) \mapsto d_{m+n-k+1}(u)^{-1}, \quad k=1, \ldots, n+m .
$$

Proof. The entries of the inverse matrix $Z^{-1}(u)$ are found from the decomposition $Z^{-1}(u)=$ $E^{-1}(u) D^{-1}(u) F^{-1}(u)$ so that

$$
z_{i^{\prime} j^{\prime}}^{\prime}(u)=\sum_{k \leqslant i, j} e_{i^{\prime} k^{\prime}}^{\prime}(u) d_{k^{\prime}}(u)^{-1} f_{k^{\prime} j^{\prime}}^{\prime}(u),
$$

where $e_{i j}^{\prime}(u)$ and $f_{i j}^{\prime}(u)$ denote the entries of the matrices $E^{-1}(u)$ and $F^{-1}(u)$, respectively, and we set $i^{\prime}=m+n-i+1$. On the other hand, the entries of $Y(u)$ are found by

$$
y_{i j}(u)=\sum_{k \leqslant i, j} f_{i k}^{\circ}(u) d_{k}^{\circ}(u) e_{k j}^{\circ}(u) .
$$

Hence, by (2.25) we have

$$
\zeta\left(d_{1}^{\circ}(u)\right)=\zeta\left(y_{11}(u)\right)=z_{1^{\prime} 1^{\prime}}^{\prime}(u)=d_{1^{\prime}}(u)^{-1} .
$$


Now, comparing (2.27) and (2.28), and arguing by induction we find that

$$
\zeta: f_{i k}^{\circ}(u) \mapsto e_{i^{\prime} k^{\prime}}^{\prime}(u), \quad e_{k j}^{\circ}(u) \mapsto f_{k^{\prime} j^{\prime}}^{\prime}(u), \quad d_{k}^{\circ}(u) \mapsto d_{k^{\prime}}(u)^{-1},
$$

as required.

Theorem 2.11. The Berezinian Ber $Z(u)$ admits the quasideterminant factorization in the superalgebra $\widehat{\mathcal{M}}_{m \mid n}[[u]]$ :

$$
\operatorname{Ber} Z(u)=d_{1}(u) \ldots d_{m}(u) d_{m+1}^{-1}(u) \ldots d_{m+n}^{-1}(u) .
$$

Proof. The upper-left $m \times m$ submatrix of $Z(u)$ is a matrix with even entries satisfying (1.1). A quasideterminant decomposition of the corresponding determinant was obtained in [4, Lemma 8] and it takes the form

$$
\sum_{\sigma \in \mathfrak{S}_{m}} \operatorname{sgn} \sigma \cdot z_{\sigma(1) 1}(u) \ldots z_{\sigma(m) m}(u)=d_{1}(u) \ldots d_{m}(u) .
$$

The second factor on the right hand side of (2.17) is the image of the determinant

$$
\sum_{\tau \in \mathfrak{S}_{n}} \operatorname{sgn} \tau \cdot y_{n, \tau(n)}(u) \ldots y_{1, \tau(1)}(u) \in \widehat{\mathcal{M}}_{n \mid m}^{\circ}[[u]]
$$

under the isomorphism (2.25). However, the upper-left $n \times n$ submatrix of $Y(u)$ is a matrix with even entries whose transpose satisfies (1.1). The corresponding quasideterminant decomposition is proved in the same way as (2.29) (see [4]), so that

$$
\sum_{\tau \in \mathfrak{S}_{n}} \operatorname{sgn} \tau \cdot y_{n, \tau(n)}(u) \ldots y_{1, \tau(1)}(u)=d_{n}^{\circ}(u) \ldots d_{1}^{\circ}(u) .
$$

By Lemma 2.10, the image of this determinant under $\zeta$ is $d_{m+1}^{-1}(u) \ldots d_{m+n}^{-1}(u)$.

An alternative factorization of the Berezinian involving different quasideterminants is provided by Corollary 2.16 below.

Remark 2.12. (i) In the super-commutative specialization the quasideterminant decompositions of the Berezinian given in Theorem 2.11 and in Corollary 2.16 below turn into the decompositions originally found by I. Gelfand and V. Retakh; cf. [14, Theorem 3.8.1].

(ii) The quantum Berezinian Ber $T(u)$ of the generator matrix of the Yangian for the Lie superalgebra $\mathfrak{g l}_{m \mid n}$ was introduced by Nazarov [37]. The quasideterminant decomposition of Ber $T(u)$ found by Gow [18, Theorem 1] can be obtained as a particular case of Theorem 2.11 by taking $Z(u)=e^{-\partial_{u}} T(u)$. The latter is a Manin matrix which follows easily from the defining relations of the Yangian; cf. [3, 4]. Hence, all identities for the Berezinian of the matrix $Z(u)$ obtained in this paper imply the corresponding counterparts for $\operatorname{Ber} T(u)$. 


\subsection{Berezinian identities}

Consider the Berezinian Ber $(1+u Z)$, where $Z$ is a Manin matrix; see Definition 2.6. The expression $\operatorname{Ber}(1+u Z)$ is a formal power series in $u$ with coefficients in the right quantum superalgebra $\mathcal{M}_{m \mid n}$. The next theorem provides some identities for the coefficients of this series, including a noncommutative analogue of the Newton identities (2.32); cf. [3], 27], [38].

Theorem 2.13. We have the identities

$$
\begin{aligned}
\operatorname{Ber}(1+u Z) & =\sum_{k=0}^{\infty} u^{k} \operatorname{str}_{1, \ldots, k} A_{k} Z_{1} \ldots Z_{k}, \\
{[\operatorname{Ber}(1-u Z)]^{-1} } & =\sum_{k=0}^{\infty} u^{k} \operatorname{str}_{1, \ldots, k} H_{k} Z_{1} \ldots Z_{k}, \\
{[\operatorname{Ber}(1+u Z)]^{-1} \partial_{u} \operatorname{Ber}(1+u Z) } & =\sum_{k=0}^{\infty}(-u)^{k} \operatorname{str} Z^{k+1} .
\end{aligned}
$$

Proof. Due to the MacMahon Master Theorem (Theorem 2.2), identities (2.30) and (2.31) are equivalent. Moreover, (2.30) is clear for $n=0$ as the Berezinian turns into a determinant. We will be proving (2.31) by induction on $n$, assuming that $n \geqslant 1$. Let $\widetilde{Z}$ be the matrix obtained from $Z$ by deleting the row and column $m+n$. Set

$$
h_{k}(Z)=\operatorname{str}_{1, \ldots, k} H_{k} Z_{1} \ldots Z_{k}=\operatorname{str}_{1, \ldots, k} Z_{1} \ldots Z_{k} H_{k} ;
$$

the second equality holds by the cyclic property of the supertrace. Applying Theorem 2.11 we derive that

$$
\operatorname{Ber}(1-u Z)=\operatorname{Ber}(1-u \widetilde{Z})\left[(1-u Z)^{-1}\right]_{m+n, m+n}
$$

and so

$$
\left[(1-u Z)^{-1}\right]_{m+n, m+n}[\operatorname{Ber}(1-u Z)]^{-1}=[\operatorname{Ber}(1-u \widetilde{Z})]^{-1} .
$$

Hence, (2.31) will follow if we show that

$$
\left[(1-u Z)^{-1}\right]_{m+n, m+n} \cdot \sum_{k=0}^{\infty} u^{k} h_{k}(Z)=\sum_{k=0}^{\infty} u^{k} h_{k}(\widetilde{Z}),
$$

or, equivalently, that for any $r \geqslant 1$ we have

$$
\sum_{k+l=r}\left(Z^{k}\right)_{m+n, m+n} \cdot h_{l}(Z)=h_{r}(\widetilde{Z}) .
$$

In order to verify (2.33) we use a relation in the superalgebra (2.1),

$$
Z_{1}^{k}=\operatorname{str}_{2, \ldots, k} Z_{1} \ldots Z_{k} P_{k-1, k} \ldots P_{23} P_{12},
$$

which follows easily by induction with the use of the relation $\operatorname{str}_{2} Z_{2} P_{12}=Z_{1}$. The next lemma is a Manin matrix version of the corresponding identities obtained in [24]. 
Lemma 2.14. We have the identity

$$
\sum_{k=1}^{r} Z_{1}^{k} h_{r-k}(Z)=r \operatorname{str}_{2, \ldots, r} Z_{1} \ldots Z_{r} H_{r}
$$

Proof. By (2.34), the left hand side can be written as

$$
\sum_{k=1}^{r} \operatorname{str}_{2, \ldots, r} Z_{1} \ldots Z_{r} H_{\{k+1, \ldots, r\}} P_{k-1, k} \ldots P_{23} P_{12}
$$

Write

$$
H_{\{k+1, \ldots, r\}}=(r-k+1) H_{\{k, \ldots, r\}}-(r-k) H_{\{k+1, \ldots, r\}} P_{k, k+1} H_{\{k+1, \ldots, r\}} .
$$

By the second relation of Proposition 2.1 and the cyclic property of the supertrace, we have

$$
\begin{aligned}
& \operatorname{str}_{2, \ldots, r} Z_{1} \ldots Z_{r} H_{\{k+1, \ldots, r\}} P_{k, k+1} H_{\{k+1, \ldots, r\}} P_{k-1, k} \ldots P_{23} P_{12} \\
= & \operatorname{str}_{2, \ldots, r} H_{\{k+1, \ldots, r\}} Z_{1} \ldots Z_{r} H_{\{k+1, \ldots, r\}} P_{k, k+1} P_{k-1, k} \ldots P_{23} P_{12} \\
= & \operatorname{str}_{2, \ldots, r} Z_{1} \ldots Z_{r} H_{\{k+1, \ldots, r\}} P_{k, k+1} \ldots P_{23} P_{12} .
\end{aligned}
$$

Hence, (2.36) takes the form of a telescoping sum which simplifies to become the right hand side of (2.35).

Taking into account Lemma 2.14, we can represent (2.33) in the equivalent form

$$
\operatorname{str}_{1, \ldots, r} Z_{1} \ldots Z_{r} H_{r}+r\left[\operatorname{str}_{2, \ldots, r} Z_{1} \ldots Z_{r} H_{r}\right]_{m+n, m+n}=\operatorname{str}_{1, \ldots, r} \widetilde{Z}_{1} \ldots \widetilde{Z}_{r} H_{r} .
$$

Write

$$
Z_{1} \ldots Z_{r} H_{r}=\sum e_{i_{1} j_{1}} \otimes \ldots \otimes e_{i_{r} j_{r}} \otimes z_{j_{1}, \ldots, j_{r}}^{i_{1}, \ldots, i_{r}}
$$

summed over all indices $i_{a}, j_{a} \in\{1, \ldots, m+n\}$, where the $z_{j_{1}, \ldots, j_{r}}^{i_{1}, \ldots, j_{r}}$ are certain elements of the right quantum superalgebra $\mathcal{M}_{m \mid n}$. Then

$$
\operatorname{str}_{1, \ldots, r} Z_{1} \ldots Z_{r} H_{r}=\sum z_{i_{1}, \ldots, i_{r}}^{i_{1}, \ldots, i_{r}}(-1)^{\bar{\tau}_{1}+\cdots+\bar{\imath}_{r}} .
$$

We have the following analogue of (2.11) which is verified in the same way:

$$
z_{i_{1} \ldots i_{a} i_{a+1} \ldots i_{k}}^{i_{1} \ldots i_{a} i_{a+1} \ldots i_{k}}=z_{i_{1} \ldots i_{a} i_{a+1} \ldots i_{k}}^{i_{1} \ldots i_{i} i_{a+1} \ldots i_{k}}(-1)^{\bar{a}_{a}}
$$

while the counterpart of (2.12) has exactly the same form. Hence, the index $m+n$ may occur at most once amongst the summation indices $i_{1}, \ldots, i_{r}$. The sum in (2.38) over the indices restricted to $i_{1}, \ldots, i_{r} \in\{1, \ldots, m+n-1\}$ coincides with the expression $\operatorname{str}_{1, \ldots, r} \widetilde{Z}_{1} \ldots \widetilde{Z}_{r} H_{r}$, while the sum over the multisets of indices containing $m+n$ equals $-r\left[\operatorname{str}_{2, \ldots, r} Z_{1} \ldots Z_{r} H_{r}\right]_{m+n, m+n}$, thus proving (2.37). This completes the proof of (2.31). 
Now we prove the Newton identity (2.32) which can be written in the equivalent form

$$
-\partial_{u}[\operatorname{Ber}(1+u Z)]^{-1}=\sum_{k=0}^{\infty}(-u)^{k} \operatorname{str} Z^{k+1} \cdot[\operatorname{Ber}(1+u Z)]^{-1} .
$$

Equating the coefficients of the same powers of $u$ we can also write this as

$$
\sum_{k=1}^{r}\left(\operatorname{str} Z^{k}\right) h_{r-k}(Z)=r h_{r}(Z), \quad r=1,2, \ldots
$$

However, this relation is immediate from Lemma 2.14 by taking the supertrace $\operatorname{str}_{1}$ over the first copy of the superalgebra End $\mathbb{C}^{m \mid n}$.

Remark 2.15. (i) In the case $n=0$ we thus get a new proof of the Newton identities for Manin matrices based on Lemma 2.14, cf. [3]. This argument essentially follows [24].

(ii) Relations of Theorem 2.13 imply that the coefficients of the powers of $u$ in the expansions (2.30), (2.31) and (2.32) can be regarded as respective specializations of the noncommutative elementary, complete and power sums symmetric functions of the first kind; see [15]. It would be interesting to find direct specializations of the other types of the noncommutative symmetric functions, in particular, the ribbon Schur functions.

Using Theorem 2.13 we can obtain alternative expressions for the Berezinians Ber $Z(u)$ and Ber $(1+u Z)$ and different quasideterminant factorizations; cf. Definition [2.6 and Theorem 2.11,

Corollary 2.16. The following relations hold

$$
\begin{aligned}
{[\operatorname{Ber} Z(u)]^{-1} } & =\sum_{\sigma \in \mathfrak{S}_{m}} \operatorname{sgn} \sigma \cdot z_{\sigma(1) 1}^{\prime}(u) \ldots z_{\sigma(m) m}^{\prime}(u) \\
& \times \sum_{\tau \in \mathfrak{S}_{n}} \operatorname{sgn} \tau \cdot z_{m+1, m+\tau(1)}(u) \ldots z_{m+n, m+\tau(n)}(u)
\end{aligned}
$$

and

$$
[\operatorname{Ber} Z(u)]^{-1}=\bar{d}_{1}^{-1}(u) \ldots \bar{d}_{m}^{-1}(u) \bar{d}_{m+1}(u) \ldots \bar{d}_{m+n}(u),
$$

where $\bar{d}_{i}(u)$ is the quasideterminant

$$
\bar{d}_{i}(u)=\left|\begin{array}{ccc}
z_{i i}(u) & \ldots & z_{i, m+n}(u) \\
\vdots & \ddots & \vdots \\
z_{m+n, i}(u) & \ldots & z_{m+n, m+n}(u)
\end{array}\right| .
$$

In particular, the corresponding relations hold for the Berezinian Ber $(1+u Z)$. 
Proof. The arguments are quite similar to those used in the proof of Theorems 2.11 and2.13 so we only sketch the main steps. We show first that the right hand side of (2.39) coincides with the product of quasideterminants on the right hand side of (2.40). Using the same calculation as in [4, Lemma 8], we obtain the following quasideterminant decomposition:

$$
\sum_{\tau \in \mathfrak{S}_{n}} \operatorname{sgn} \tau \cdot z_{m+1, m+\tau(1)}(u) \ldots z_{m+n, m+\tau(n)}(u)=\bar{d}_{m+1}(u) \ldots \bar{d}_{m+n}(u) .
$$

Furthermore, the determinant

$$
\sum_{\sigma \in \mathfrak{S}_{m}} \operatorname{sgn} \sigma \cdot z_{\sigma(1) 1}^{\prime}(u) \ldots z_{\sigma(m) m}^{\prime}(u)
$$

coincides with the image of a certain determinant of a submatrix of $Y(u)$ under the isomorphism (2.25) which leads to the desired factorization via a natural dual analogue of Lemma 2.10,

Observe that the matrix $Z(u)$ can be written as $Z(u)=1+\widetilde{Z}(u)$ and $\widetilde{Z}(u)$ satisfies (1.9). It is therefore sufficient to verify relations (2.39) and (2.40) for matrices of the form $Z(u)=1+u Z$, where $Z$ satisfies (1.8). Since we have verified that the right hand sides of (2.39) and (2.40) coincide, it is enough to show that (2.40) holds. We will use induction on $m$, assuming that $m \geqslant 1$. Let $\bar{Z}$ be the matrix obtained from $Z$ by deleting the row and column 1 . Then we need to verify that

$$
[\operatorname{Ber}(1+u Z)]^{-1}=\left[(1+u Z)^{-1}\right]_{11}[\operatorname{Ber}(1+u \bar{Z})]^{-1},
$$

or, equivalently,

$$
\operatorname{Ber}(1+u Z) \cdot\left[(1+u Z)^{-1}\right]_{11}=\operatorname{Ber}(1+u \bar{Z})
$$

Set

$$
\sigma_{k}(Z)=\operatorname{str}_{1, \ldots, k} A_{k} Z_{1} \ldots Z_{k} .
$$

Due to (2.30), the relation (2.41) will follow if we show that for any $r \geqslant 1$

$$
\sum_{k+l=r} \sigma_{k}(Z) \cdot\left(Z^{l}\right)_{11}=\sigma_{r}(\bar{Z})
$$

However, this follows by the same argument as for the proof of (2.33).

\section{Sugawara operators for $\widehat{\mathfrak{g l}}_{m \mid n}$}

Consider the Lie superalgebra $\widehat{\mathfrak{g l}}_{m \mid n} \oplus \mathbb{C} \tau$ with its commutation relations (1.15) and (1.16). We assume that $m$ and $n$ are nonnegative integers. Recall also that $V=V_{\kappa}\left(\mathfrak{g l}_{m \mid n}\right)$ is the affine vertex algebra at the level $\kappa \in \mathbb{C}$. This means that $V$ is equipped with the additional 
data $(Y, D, 1)$, where $1 \in V$ is the vacuum vector, the state-field correspondence $Y$ is a map

$$
Y: V \rightarrow \text { End } V\left[\left[z, z^{-1}\right]\right]
$$

the infinitesimal translation $D$ is an operator $D: V \rightarrow V$. These data satisfy the vertex algebra axioms; see e.g. [12], [26]. For $a \in V$ we write

$$
Y(a, z)=\sum_{r \in \mathbb{Z}} a_{(r)} z^{-r-1}, \quad a_{(r)} \in \text { End } V .
$$

In particular, for all $a, b \in V$ we have $a_{(r)} b=0$ for $r \gg 0$. The span in End $V$ of all Fourier coefficients $a_{(r)}$ of all vertex operators $Y(a, z)$ is a Lie superalgebra $\mathrm{U}_{\kappa}\left(\widehat{\mathfrak{g l}}_{m \mid n}\right)_{\text {loc }}$ with the super-commutator

$$
\left[a_{(r)}, b_{(s)}\right]=\sum_{k \geqslant 0}\left(\begin{array}{l}
r \\
k
\end{array}\right)\left(a_{(k)} b\right)_{(r+s-k)},
$$

which is called the local completion of the quotient of the universal enveloping algebra $\mathrm{U}\left(\widehat{\mathfrak{g l}}_{m \mid n}\right)$ by the ideal generated by $K-\kappa$; see [12, Sec. 3.5].

The translation operator is determined by

$$
D: 1 \mapsto 0 \quad \text { and } \quad\left[D, e_{i j}[r]\right]=-r e_{i j}[r-1] .
$$

The state-field correspondence $Y$ is defined by setting $Y(1, z)=\mathrm{id}$,

$$
Y\left(e_{i j}[-1], z\right)=e_{i j}(z):=\sum_{r \in \mathbb{Z}} e_{i j}[r] z^{-r-1},
$$

and then extending the map to the whole of $V$ with the use of normal ordering. Namely, the normally ordered product of homogeneous fields

$$
a(z)=\sum_{r \in \mathbb{Z}} a_{(r)} z^{-r-1} \quad \text { and } \quad b(w)=\sum_{r \in \mathbb{Z}} b_{(r)} w^{-r-1}
$$

is the formal power series

$$
: a(z) b(w):=a(z)_{+} b(w)+(-1)^{\operatorname{deg} a \operatorname{deg} b} b(w) a(z)_{-},
$$

where

$$
a(z)_{+}=\sum_{r<0} a_{(r)} z^{-r-1} \quad \text { and } \quad a(z)_{-}=\sum_{r \geqslant 0} a_{(r)} z^{-r-1} .
$$

This definition extends to an arbitrary number of fields with the convention that the normal ordering is read from right to left. Then

$$
Y\left(e_{i_{1} j_{1}}\left[-r_{1}-1\right] \ldots e_{i_{m} j_{m}}\left[-r_{m}-1\right], z\right)=\frac{1}{r_{1} ! \ldots r_{m} !}: \partial_{z}^{r_{1}} e_{i_{1} j_{1}}(z) \ldots \partial_{z}^{r_{m}} e_{i_{m} j_{m}}(z):
$$


As defined in the Introduction, the center of the vertex algebra $V_{n-m}\left(\mathfrak{g l}_{m \mid n}\right)$ at the critical level $\kappa=n-m$ is

$$
\mathfrak{z}\left(\widehat{\mathfrak{g l}}_{m \mid n}\right)=\left\{b \in V_{n-m}\left(\mathfrak{g l}_{m \mid n}\right) \mid \mathfrak{g l}_{m \mid n}[t] b=0\right\}
$$

which can be identified with a commutative subalgebra of $\mathrm{U}\left(t^{-1} \mathfrak{g l}_{m \mid n}\left[t^{-1}\right]\right)$. Elements of $\mathfrak{z}\left(\widehat{\mathfrak{g l}}_{m \mid n}\right)$ are called Segal-Sugawara vectors. Due to the commutator formula (3.1), if $b \in \mathfrak{z}\left(\widehat{\mathfrak{g l}}_{m \mid n}\right)$, then all Fourier coefficients of the corresponding field $b(z)=Y(b, z)$ belong to the center of the Lie superalgebra $\mathrm{U}_{\kappa}\left(\widehat{\mathfrak{g l}}_{m \mid n}\right)_{\text {loc }}$. These Fourier coefficients are called the Sugawara operators for $\widehat{\mathfrak{g l}}_{m \mid n}$. In particular, they commute with the elements of $\widehat{\mathfrak{g l}}_{m \mid n}$ and thus form a commuting family of $\widehat{\mathfrak{g l}}_{m \mid n}$-endomorphisms of Verma modules over $\widehat{\mathfrak{g l}}_{m \mid n}$ at the critical level; cf. [7], [16], 23]. We will apply the results of Sec. 2 to construct several families of Sugawara operators for $\widehat{\mathfrak{g l}}_{m \mid n}$.

\subsection{Segal-Sugawara vectors}

Consider the square matrix

$$
\tau+\widehat{E}[-1]=\left[\delta_{i j} \tau+e_{i j}[-1](-1)^{\bar{\imath}}\right]
$$

with the entries in the universal enveloping algebra for $\widehat{\mathfrak{g l}}_{m \mid n} \oplus \mathbb{C} \tau$. The following observation will play a key role in what follows.

Lemma 3.1. The matrix $\tau+\widehat{E}[-1]$ is a Manin matrix.

Proof. We have

$$
\begin{aligned}
{\left[\delta_{i j} \tau+e_{i j}[-1](-1)^{\bar{\imath}},\right.} & \left.\delta_{k l} \tau+e_{k l}[-1](-1)^{\bar{k}}\right] \\
& =\delta_{i j} e_{k l}[-2](-1)^{\bar{k}}-\delta_{k l} e_{i j}[-2](-1)^{\bar{\imath}} \\
& +\delta_{k j} e_{i l}[-2](-1)^{\bar{\imath}+\bar{k}}-\delta_{i l} e_{k j}[-2](-1)^{(\bar{\imath}+\bar{\jmath})(\bar{k}+\bar{l})+\bar{\imath}+\bar{k}}
\end{aligned}
$$

This expression remains unchanged after swapping $i$ and $k$ and multiplying by $(-1)^{\bar{\imath} \bar{\jmath}+\bar{\imath} \bar{k}+\bar{\jmath} \bar{k}}$. Thus, the matrix elements of $\tau+\widehat{E}[-1]$ satisfy (1.2).

Theorem 3.2. For any $k \geqslant 0$ all coefficients $s_{k l}$ in the expansion

$$
\operatorname{str}(\tau+\widehat{E}[-1])^{k}=s_{k 0} \tau^{k}+s_{k 1} \tau^{k-1}+\cdots+s_{k k}
$$

are Segal-Sugawara vectors. 
Proof. It is sufficient to verify that for all $i, j$

$$
e_{i j}[0] \operatorname{str}(\tau+\widehat{E}[-1])^{k}=e_{i j}[1] \operatorname{str}(\tau+\widehat{E}[-1])^{k}=0
$$

in the $\widehat{\mathfrak{g l}}_{m \mid n}$-module $V_{n-m}\left(\mathfrak{g l}_{m \mid n}\right) \otimes \mathbb{C}[\tau]$. We will employ matrix notation of Sec. 2.1 and consider the tensor product superalgebra

$$
\text { End } \mathbb{C}^{m \mid n} \otimes \ldots \otimes \text { End } \mathbb{C}^{m \mid n} \otimes \mathrm{U}
$$

with $k+1$ copies of End $\mathbb{C}^{m \mid n}$ labeled by $0,1, \ldots, k$, where $\mathrm{U}$ stands for the universal enveloping algebra $\mathrm{U}\left(\widehat{\mathfrak{g l}}_{m \mid n} \oplus \mathbb{C} \tau\right)$. Set $T=\tau+\widehat{E}[-1]$ and for any integer $r$ introduce the matrix $\widehat{E}[r]=\left[e_{i j}[r](-1)^{\bar{\imath}}\right]$. Relations $(\underline{3.6})$ can now be written in the equivalent form

$$
\widehat{E}_{0}[0] \operatorname{str}_{1} T_{1}^{k}=0 \quad \text { and } \quad \widehat{E}_{0}[1] \operatorname{str}_{1} T_{1}^{k}=0
$$

modulo the left ideal of $\mathrm{U}$ generated by $\mathfrak{g l}_{m \mid n}[t]$ and $K-n+m$ (or by $K^{\prime}-1$ in terms of the re-scaled central element $\left.K^{\prime}=K /(n-m)\right)$. In order to verity them, note that by the commutation relations in the Lie superalgebra $\widehat{\mathfrak{g l}}_{m \mid n} \oplus \mathbb{C} \tau$ we have

$$
\begin{aligned}
& {\left[\widehat{E}_{0}[0], T_{1}\right]=P_{01} T_{1}-T_{1} P_{01},} \\
& {\left[\widehat{E}_{0}[1], T_{1}\right]=\widehat{E}_{0}[0]+P_{01} \widehat{E}_{1}[0]-\widehat{E}_{1}[0] P_{01}+K P_{01}+\frac{K}{n-m} ;}
\end{aligned}
$$

the last two terms in (3.9) (and throughout the argument below) should be re-written in terms of $K^{\prime}$ in the case $m=n$. The following identity is well-known:

$$
\left[\widehat{E}_{0}[0], T_{1}^{k}\right]=P_{01} T_{1}^{k}-T_{1}^{k} P_{01}, \quad k=0,1,2, \ldots,
$$

and it follows immediately from (3.8):

$$
\left[\widehat{E}_{0}[0], T_{1}^{k}\right]=\sum_{r=1}^{k} T_{1}^{r-1}\left[\widehat{E}_{0}[0], T_{1}\right] T_{1}^{k-r}=\sum_{r=1}^{k} T_{1}^{r-1}\left[P_{01}, T_{1}\right] T_{1}^{k-r}=\left[P_{01}, T_{1}^{k}\right] .
$$

Now the first relation in (3.7) follows by taking the supertrace $\operatorname{str}_{1}$ on both sides of (3.10).

For the proof of the second relation in (3.7), suppose first that $m \neq n$ and use (3.9) to write

$$
\left[\widehat{E}_{0}[1], T_{1}^{k}\right]=\sum_{i=1}^{k} T_{1}^{i-1}\left(\widehat{E}_{0}[0]+P_{01} \widehat{E}_{1}[0]-\widehat{E}_{1}[0] P_{01}+K P_{01}-\frac{K}{m-n}\right) T_{1}^{k-i}
$$

Applying (3.10) and the relation $\operatorname{str}_{2} T_{2}^{k-i} P_{12}=T_{1}^{k-i}$ we can rewrite (3.11) modulo the left ideal of $\mathrm{U}$ generated by $\mathfrak{g l}_{m \mid n}[t]$ as

$$
\begin{aligned}
{\left[\widehat{E}_{0}[1], T_{1}^{k}\right] } & =\sum_{i=1}^{k}\left(T_{1}^{i-1}\left[P_{01}, T_{1}^{k-i}\right]+K T_{1}^{i-1} P_{01} T_{1}^{k-i}\right)-\frac{k K}{m-n} T_{1}^{k-1} \\
& +\operatorname{str}_{2} \sum_{i=1}^{k} T_{1}^{i-1}\left(P_{01} \widehat{E}_{1}[0]-\widehat{E}_{1}[0] P_{01}\right) T_{2}^{k-i} P_{12} .
\end{aligned}
$$


Now transform the last summand using (3.10) to get

$$
\begin{aligned}
& \operatorname{str}_{2} \sum_{i=1}^{k} T_{1}^{i-1}\left(P_{01} \widehat{E}_{1}[0]-\widehat{E}_{1}[0] P_{01}\right) T_{2}^{k-i} P_{12} \\
= & \operatorname{str}_{2} \sum_{i=1}^{k} T_{1}^{i-1} P_{01}\left[P_{12}, T_{2}^{k-i}\right] P_{12}-\operatorname{str}_{2} \sum_{i=1}^{k} T_{1}^{i-1}\left[P_{12}, T_{2}^{k-i}\right] P_{01} P_{12} .
\end{aligned}
$$

Taking into account the relation $\operatorname{str}_{2} P_{02}=1$, we can simplify this to

$$
\sum_{i=1}^{k-1}\left((m-n) T_{1}^{i-1} P_{01} T_{1}^{k-i}-T_{1}^{i-1} P_{01} \operatorname{str} T^{k-i}+T_{1}^{i-1} T_{0}^{k-i}\right)-(k-1) T_{1}^{k-1} .
$$

Combining all the terms and taking the supertrace $\operatorname{str}_{1}$ in (3.11) we derive

$$
\begin{aligned}
{\left[\widehat{E}_{0}[1], \operatorname{str}_{1} T_{1}^{k}\right] } & =(K+m-n)\left(T_{0}^{k-1}-\frac{k}{m-n} \operatorname{str} T^{k-1}\right)+(K+m-n-k+2) T_{0}^{k-1} \\
& +(K+m-n+1) \operatorname{str}_{1} \sum_{i=2}^{k-1} P_{01} T_{0}^{i-1} T_{1}^{k-i}-\operatorname{str}_{1} \sum_{i=2}^{k-1}\left[T_{0}^{i-1}, T_{1}^{k-i}\right] .
\end{aligned}
$$

Finally, we use Lemmas 2.7 and 3.1 to write

$$
\sum_{i=2}^{k-1}\left[T_{0}^{i-1}, T_{1}^{k-i}\right]=\sum_{i=2}^{k-1} P_{01}\left[T_{0}^{i-1}, T_{1}^{k-i}\right]
$$

which brings the previous formula to the form

$$
\left[\widehat{E}_{0}[1], \operatorname{str}_{1} T_{1}^{k}\right]=(K+m-n)\left(2 T_{0}^{k-1}-\frac{k}{m-n} \operatorname{str} T^{k-1}+\operatorname{str}_{1} \sum_{i=2}^{k-1} P_{01} T_{0}^{i-1} T_{1}^{k-i}\right) .
$$

This expression vanishes in the vacuum module $V_{n-m}\left(\mathfrak{g l}_{m \mid n}\right)$ as $K+m-n=0$.

In the case $m=n$ the same calculation applies with the use of (3.9) rewritten in terms of $K^{\prime}=K /(n-m)$. The critical value of $K^{\prime}$ is understood as being equal to 1 .

The following corollary is immediate from Theorems 2.13 and 3.2 .

Corollary 3.3. All the coefficients $\sigma_{k l}, h_{k l}, b_{k l} \in \mathrm{U}\left(t^{-1} \mathfrak{g l}_{m \mid n}\left[t^{-1}\right]\right)$ in the expansions

$$
\begin{aligned}
\operatorname{str}_{1, \ldots, k} A_{k} T_{1} \ldots T_{k} & =\sigma_{k 0} \tau^{k}+\sigma_{k 1} \tau^{k-1}+\cdots+\sigma_{k k}, \\
\operatorname{str}_{1, \ldots, k} H_{k} T_{1} \ldots T_{k} & =h_{k 0} \tau^{k}+h_{k 1} \tau^{k-1}+\cdots+h_{k k}, \\
\operatorname{Ber}(1+u T) & =\sum_{k=0}^{\infty} \sum_{l=0}^{k} b_{k l} u^{k} \tau^{k-l}
\end{aligned}
$$

are Segal-Sugawara vectors. Moreover, $b_{k l}=\sigma_{k l}$ for all $k$ and $l$. 
Remark 3.4. (i) In the case $n=0$ the formulas of Theorem 3.2 and Corollary 3.3 reproduce the explicit constructions of Segal-Sugawara vectors for the affine Kac-Moody algebra $\widehat{\mathfrak{g l}}_{m}$; see [5] and [6]. The Berezinian Ber $(1+u T)$ turns into the column determinant $\operatorname{cdet}(1+u T)$ which is a polynomial in $u$, and the proof given in [5] deals with its leading coefficient cdet $T$. The use of the matrix techniques in the above proof of Theorem 3.2 thus provided a simpler proof of those results for $\widehat{\mathfrak{g l}}_{m}$.

(ii) Denote by $\bar{s}_{k k}, \bar{h}_{k k}$ and $\bar{b}_{k k}$ the respective images of the elements $s_{k k}, h_{k k}$ and $b_{k k}$ in the associated graded algebra gr $\mathrm{U}\left(t^{-1} \mathfrak{g l}_{m \mid n}\left[t^{-1}\right]\right) \cong \mathrm{S}\left(t^{-1} \mathfrak{g l}_{m \mid n}\left[t^{-1}\right]\right)$. Each of the families $\bar{s}_{k k}, \bar{h}_{k k}$ and $\bar{b}_{k k}$ with $k \geqslant 1$ is the image of a generating set of the algebra of invariants $\mathrm{S}\left(\mathfrak{g l}_{m \mid n}\right)^{\mathfrak{g l}_{m \mid n}}$ under the embedding $\mathrm{S}\left(\mathfrak{g l}_{m \mid n}\right) \hookrightarrow \mathrm{S}\left(t^{-1} \mathfrak{g l}_{m \mid n}\left[t^{-1}\right]\right)$ defined by the assignment $X \mapsto X[-1]$. In the case $n=0$ the families of Segal-Sugawara vectors with such a property are known as complete sets of Segal-Sugawara vectors (see [5], 7], [16], [23]) so that this terminology can be extended to the super case. It is natural to suppose that each of the families $D^{r} s_{k k}, D^{r} h_{k k}$ and $D^{r} b_{k k}$ with $r \geqslant 0$ and $k \geqslant 1$ generates the center $\mathfrak{z}\left(\widehat{\mathfrak{g l}}_{m \mid n}\right)$ of the vertex algebra $V_{n-m}\left(\mathfrak{g l}_{m \mid n}\right)$; cf. [11]. However, we do not have a proof of this conjecture. If both $m$ and $n$ are positive integers, then none of the families is algebraically independent; cf. Theorem 3.10 below.

The application of the state-field correspondence map $Y$ to the Segal-Sugawara vectors produces elements of the center of the local completion $\mathrm{U}_{n-m}\left(\widehat{\mathfrak{g l}}_{m \mid n}\right)_{\text {loc }}$ at the critical level $\kappa=n-m$. Hence, Theorem 3.2 and Corollary 3.3 provide explicit formulas for the corresponding Sugawara operators for $\widehat{\mathfrak{g l}}_{m \mid n}$.

Recall the matrix $\widehat{E}(z)=\left[e_{i j}(z)(-1)^{\bar{\imath}}\right]$, where the fields $e_{i j}(z)$ are defined in (3.3). Set $T(z)=\partial_{z}+\widehat{E}(z)$, where $\partial_{z}$ is understood as a scalar matrix of size $m+n$.

Corollary 3.5. All Fourier coefficients of the fields $s_{k l}(z), b_{k l}(z), \sigma_{k l}(z)$ and $h_{k l}(z)$ defined by the decompositions

$$
\begin{aligned}
: \operatorname{str} T(z)^{k}: & =s_{k 0}(z) \partial_{z}^{k}+s_{k 1}(z) \partial_{z}^{k-1}+\cdots+s_{k k}(z), \\
: \operatorname{Ber}(1+u T(z)): & =\sum_{k=0}^{\infty} \sum_{l=0}^{k} b_{k l}(z) u^{k} \partial_{z}^{k-l} \\
: \operatorname{str}_{1, \ldots, k} A_{k} T_{1}(z) \ldots T_{k}(z): & =\sigma_{k 0}(z) \partial_{z}^{k}+\sigma_{k 1}(z) \partial_{z}^{k-1}+\cdots+\sigma_{k k}(z), \\
: \operatorname{str}_{1, \ldots, k} H_{k} T_{1}(z) \ldots T_{k}(z): & =h_{k 0}(z) \partial_{z}^{k}+h_{k 1}(z) \partial_{z}^{k-1}+\cdots+h_{k k}(z)
\end{aligned}
$$

are Sugawara operators for $\widehat{\mathfrak{g l}}_{m \mid n}$. Moreover, $\sigma_{k l}(z)=b_{k l}(z)$ for all $k$ and $l$.

\subsection{Commutative subalgebras and super Gaudin Hamiltonians}

By the vacuum axiom of a vertex algebra, the application of any of the fields $s_{k l}(z), b_{k l}(z)$, $\sigma_{k l}(z)$ and $h_{k l}(z)$ introduced in Corollary 3.5 to the vacuum vector yields formal power 
series in $z$ with coefficients in the universal enveloping algebra $\mathrm{U}\left(t^{-1} \mathfrak{g l}_{m \mid n}\left[t^{-1}\right]\right)$. Moreover, since the Fourier coefficients of the fields belong to the center of the local completion $\mathrm{U}_{n-m}\left(\widehat{\mathfrak{g l}}_{m \mid n}\right)_{\text {loc }}$, all coefficients of the power series will belong to the center $\mathfrak{z}\left(\widehat{\mathfrak{g l}}_{m \mid n}\right)$ of the vertex algebra $V_{n-m}\left(\mathfrak{g l}_{m \mid n}\right)$. In particular, these coefficients generate a commutative subalgebra of $\mathrm{U}\left(t^{-1} \mathfrak{g l}_{m \mid n}\left[t^{-1}\right]\right)$. Explicitly, they can be given by the same expansions as in Corollary 3.5 by omitting the normal ordering signs and by replacing $T(z)$ with the matrix $T(z)_{+}=\partial_{z}+\widehat{E}(z)_{+}$with $\widehat{E}(z)_{+}=\left[(-1)^{\bar{\imath}} e_{i j}(z)_{+}\right]$and

$$
e_{i j}(z)_{+}=\sum_{r=0}^{\infty} e_{i j}[-r-1] z^{r} .
$$

Now we produce the corresponding families of commuting elements of $\mathrm{U}\left(\mathfrak{g l}_{m \mid n}[t]\right)$. The commutation relations of the Lie superalgebra $t^{-1} \mathfrak{g l}_{m \mid n}\left[t^{-1}\right]$ can be written in terms of the series (3.12) as

$$
\begin{aligned}
(z-w)\left[e_{i j}(z)_{+}, e_{k l}(w)_{+}\right] & =\delta_{k j}\left(e_{i l}(z)_{+}-e_{i l}(w)_{+}\right) \\
& -\delta_{i l}\left(e_{k j}(z)_{+}-e_{k j}(w)_{+}\right)(-1)^{(\bar{\imath}+\bar{\jmath})(\bar{k}+\bar{l})} .
\end{aligned}
$$

Setting

$$
e_{i j}(z)_{-}=\sum_{r=0}^{\infty} e_{i j}[r] z^{-r-1}
$$

we find that

$$
\begin{aligned}
(z-w)\left[e_{i j}(z)_{-}, e_{k l}(w)_{-}\right]= & -\delta_{k j}\left(e_{i l}(z)_{-}-e_{i l}(w)_{-}\right) \\
& +\delta_{i l}\left(e_{k j}(z)_{-}-e_{k j}(w)_{-}\right)(-1)^{(\bar{\imath}+\bar{\jmath})(\bar{k}+\bar{l})} .
\end{aligned}
$$

The commuting families of elements of $\mathrm{U}\left(t^{-1} \mathfrak{g l}_{m \mid n}\left[t^{-1}\right]\right)$ obtained by the application of Corollary 3.5 are expressed as coefficients of certain differential polynomials in the series $e_{i j}(z)_{+}$(i.e., polynomials in $\left.\partial_{z}^{k} e_{i j}(z)_{+}\right)$. Therefore, comparing (3.13) and (3.14), we can conclude that the same differential polynomials with the $e_{i j}(z)+$ respectively replaced by $-e_{i j}(z)_{-}$, generate a commutative subalgebra of $\mathrm{U}\left(\mathfrak{g l}_{m \mid n}[t]\right)$. Indeed, suppose that $A(z)$ is a differential polynomial in the $e_{i j}(z)_{+}$and $B(w)$ is a differential polynomial in the $e_{i j}(w)_{+}$. A relation of the form $[A(z), B(w)]=0$ is a consequence of the commutation relations between the series (3.13) where the actual expansions of the $e_{i j}(z)_{+}$and $e_{i j}(w)_{+}$as power series in $z$ and $w$ need not be used. This relies on the easily verified property that given any total ordering on the set of series $\partial_{z}^{k} e_{i j}(z)_{+}$, the corresponding ordered monomials are linearly independent over the polynomial ring $\mathbb{C}[z]$. Thus we arrive at the following corollary, where we use the notation

$$
L(z)=\partial_{z}-\widehat{E}(z)_{-}, \quad \widehat{E}(z)_{-}=\left[(-1)^{\bar{\imath}} e_{i j}(z)_{-}\right]
$$


Corollary 3.6. All coefficients of the series $S_{k l}(z), B_{k l}(z), \Sigma_{k l}(z)$ and $H_{k l}(z)$ defined by the decompositions

$$
\begin{aligned}
\operatorname{str} L(z)^{k} & =S_{k 0}(z) \partial_{z}^{k}+S_{k 1}(z) \partial_{z}^{k-1}+\cdots+S_{k k}(z), \\
\operatorname{Ber}(1+u L(z)) & =\sum_{k=0}^{\infty} \sum_{l=0}^{k} B_{k l}(z) u^{k} \partial_{z}^{k-l}, \\
\operatorname{str}_{1, \ldots, k} A_{k} L_{1}(z) \ldots L_{k}(z) & =\Sigma_{k 0}(z) \partial_{z}^{k}+\Sigma_{k 1}(z) \partial_{z}^{k-1}+\cdots+\Sigma_{k k}(z), \\
\operatorname{str}_{1, \ldots, k} H_{k} L_{1}(z) \ldots L_{k}(z) & =H_{k 0}(z) \partial_{z}^{k}+H_{k 1}(z) \partial_{z}^{k-1}+\cdots+H_{k k}(z)
\end{aligned}
$$

generate a commutative subalgebra of $\mathrm{U}\left(\mathfrak{g l}_{m \mid n}[t]\right)$. Moreover, $\Sigma_{k l}(z)=B_{k l}(z)$ for all values of $k$ and $l$.

Note also that given any set of complex parameters $\lambda_{1}, \ldots, \lambda_{m+n}$ the commutation relations (3.14) remain valid after the replacement

$$
e_{i j}(z)_{-} \mapsto \delta_{i j} \lambda_{i}+e_{i j}(z)_{-}
$$

Thus, we obtain more general families of commutative subalgebras of $\mathrm{U}\left(\mathfrak{g l}_{m \mid n}[t]\right)$.

Corollary 3.7. Given a set of complex parameters $\lambda_{1}, \ldots, \lambda_{m+n}$, all coefficients of the series $S_{k l}(z), B_{k l}(z), \Sigma_{k l}(z)$ and $H_{k l}(z)$ defined by the decompositions of Corollary 3.6 with the series $e_{i j}(z)_{\text {- replaced as in (3.15) }}$ generate a commutative subalgebra of $\mathrm{U}\left(\mathfrak{g l}_{m \mid n}[t]\right)$.

Remark 3.8. (i) A more general replacement $e_{i j}(z)_{-} \mapsto K_{i j}+e_{i j}(z)_{-}$with appropriately defined elements $K_{i j}$ preserves the commutation relations (3.14) as well, thus leading to even more general family of commutative subalgebras of $\mathrm{U}\left(\mathfrak{g l}_{m \mid n}[t]\right)$.

(ii) In the particular case $n=0$ the elements of the commutative subalgebras of $\mathrm{U}\left(\mathfrak{g l}_{m}[t]\right)$ from Corollaries 3.6 and 3.7 were originally constructed in [39]; see also [5], 6], [35], [36]. In particular, the Berezinian turns into a determinant, and generators of the commutative subalgebra are found by the decomposition of the column determinant

$$
\operatorname{cdet}\left[\begin{array}{cccc}
\partial_{z}-e_{11}(z)_{-} & -e_{12}(z)_{-} & \ldots & -e_{1 m}(z)_{-} \\
-e_{21}(z)_{-} & \partial_{z}-e_{22}(z)_{-} & \ldots & -e_{2 m}(z)_{-} \\
\vdots & \vdots & \ddots & \vdots \\
-e_{m 1}(z)_{-} & -e_{m 2}(z)_{-} & \ldots & \partial_{z}-e_{m m}(z)_{-}
\end{array}\right] .
$$

The commutative subalgebras of $\mathrm{U}\left(\mathfrak{g l}_{m \mid n}[t]\right)$ can be used to construct higher Gaudin Hamiltonians following the same scheme as in the even case; see [8], [35], [39]. More precisely, given a finite-dimensional $\mathfrak{g l}_{m \mid n}$-module $M$ and a complex number $a$ we can define the corresponding evaluation $\mathfrak{g l}_{m \mid n}[t]$-module $M_{a}$. As a vector superspace, $M_{a}$ coincides 
with $M$, while the action of the elements of the Lie superalgebra is given by $e_{i j}[r] \mapsto e_{i j} a^{r}$ for $r \geqslant 0$, or equivalently,

$$
e_{i j}(z)_{-} \mapsto \frac{e_{i j}}{z-a} .
$$

Now consider certain finite-dimensional $\mathfrak{g l}_{m \mid n}$-modules $M^{(1)}, \ldots, M^{(k)}$ and let $a_{1}, \ldots, a_{k}$ be complex parameters. Then the tensor product of the evaluation modules

$$
M_{a_{1}}^{(1)} \otimes \ldots \otimes M_{a_{k}}^{(k)}
$$

becomes a $\mathfrak{g l}_{m \mid n}[t]$-module via the iterated comultiplication map (1.20). Then the images of the matrix elements of the matrix $L(z)$ are found by

$$
\ell_{i j}(z)=\delta_{i j} \partial_{z}-(-1)^{\bar{\imath}} \sum_{r=1}^{k} \frac{e_{i j}^{(r)}}{z-a_{r}},
$$

where $e_{i j}^{(r)}$ denotes the image of $e_{i j}$ in the $\mathfrak{g l}_{m \mid n}$-module $M^{(r)}$. So replacing $L(z)$ by the matrix $\mathcal{L}(z)=\left[\ell_{i j}(z)\right]$ in the formulas of Corollary 3.6 we obtain a family of commuting operators in the module (3.16), thus producing higher Gaudin Hamiltonians associated with $\mathfrak{g l}_{m \mid n}$. In particular, such families are provided by the coefficients of the series defined by the expansion of the Berezinian $\operatorname{Ber}(1+u \mathcal{L}(z))$ and the supertrace

$$
\operatorname{str} \mathcal{L}(z)^{k}=\mathcal{S}_{k 0}(z) \partial_{z}^{k}+\mathcal{S}_{k 1}(z) \partial_{z}^{k-1}+\cdots+\mathcal{S}_{k k}(z) .
$$

The quadratic Gaudin Hamiltonian $\mathcal{H}(z)=\mathcal{S}_{22}(z)$ can be written explicitly as

$$
\mathcal{H}(z)=\sum_{r, s=1}^{k} \frac{1}{\left(z-a_{r}\right)\left(z-a_{s}\right)} \sum_{i, j} e_{i j}^{(r)} e_{j i}^{(s)}(-1)^{\bar{j}}+\sum_{r=1}^{k} \frac{1}{\left(z-a_{r}\right)^{2}} \sum_{i} e_{i i}^{(r)} .
$$

Assuming further that the parameters $a_{i}$ are all distinct and setting

$$
\mathcal{H}^{(r)}=\sum_{s \neq r} \frac{1}{a_{r}-a_{s}} \sum_{i, j} e_{i j}^{(r)} e_{j i}^{(s)}(-1)^{\bar{\jmath}}
$$

we can also write the Hamiltonian as

$$
\mathcal{H}(z)=2 \sum_{r=1}^{k} \frac{\mathcal{H}^{(r)}}{z-a_{r}}+\sum_{r=1}^{k} \frac{\Delta^{(r)}}{\left(z-a_{r}\right)^{2}},
$$

where $\Delta^{(r)}$ denotes the eigenvalue of the Casimir element $\sum e_{i j} e_{j i}(-1)^{\bar{j}}+\sum e_{i i}$ of $\mathfrak{g l}_{m \mid n}$ in the representation $M^{(r)}$; cf. [8], [29].

More general families of commuting elements in $\mathrm{U}\left(\mathfrak{g l}_{m \mid n}[t]\right)$ and higher Gaudin Hamiltonians can be constructed by using extra parameters $\lambda_{i}$ or $K_{i j}$ as in Corollary 3.7 and Remark 3.8(i). 


\subsection{Singular vectors in Verma modules}

We now recall a result from [17] and formulate it for the affine Lie superalgebra of type $A(m-1 \mid n-1)$. Consider the Lie superalgebra $\widehat{\mathfrak{g}}=\widehat{\mathfrak{g l}}_{m \mid n} \oplus \mathbb{C} d$, so that

$$
\widehat{\mathfrak{g}}=\mathfrak{g l}_{m \mid n}\left[t, t^{-1}\right] \oplus \mathbb{C} K \oplus \mathbb{C} d,
$$

with the commutation relations (1.15), where the elements $K$ and $d$ are even, $K$ is central and

$$
\left[d, e_{i j}[r]\right]=r e_{i j}[r] .
$$

Denote by $\mathfrak{n}_{-}, \mathfrak{h}$ and $\mathfrak{n}$ the subalgebras of $\mathfrak{g l}_{m \mid n}$ spanned by the lower-triangular, diagonal and upper triangular matrices, respectively. We have the triangular decomposition

$$
\widehat{\mathfrak{g}}=\widehat{\mathfrak{n}}_{-} \oplus \widehat{\mathfrak{h}} \oplus \widehat{\mathfrak{n}},
$$

where $\widehat{\mathfrak{h}}$ is the Cartan subalgebra of $\widehat{\mathfrak{g}}$ defined by

$$
\widehat{\mathfrak{h}}=\mathfrak{h} \oplus \mathbb{C} K \oplus \mathbb{C} d,
$$

while

$$
\widehat{\mathfrak{n}}_{-}=\mathfrak{n}_{-}\left[t^{-1}\right] \oplus t^{-1} \mathfrak{h}\left[t^{-1}\right] \oplus t^{-1} \mathfrak{n}\left[t^{-1}\right] \quad \text { and } \quad \widehat{\mathfrak{n}}=t \mathfrak{n}_{-}[t] \oplus t \mathfrak{h}[t] \oplus \mathfrak{n}[t]
$$

We have the direct sum decomposition

$$
\mathrm{U}\left(\widehat{\mathfrak{n}}_{-}\right)=\mathrm{U}\left(t^{-1} \mathfrak{h}\left[t^{-1}\right]\right) \oplus\left(\mathrm{U}\left(\widehat{\mathfrak{n}}_{-}\right) \mathfrak{n}_{-}\left[t^{-1}\right]+t^{-1} \mathfrak{n}\left[t^{-1}\right] \mathrm{U}\left(\widehat{\mathfrak{n}}_{-}\right)\right) .
$$

Introduce the projection to the first component in the direct sum

$$
\mathcal{H} C: \mathrm{U}\left(\widehat{\mathfrak{n}}_{-}\right) \rightarrow \mathrm{U}\left(t^{-1} \mathfrak{h}\left[t^{-1}\right]\right)
$$

so that the kernel of $\mathcal{H C}$ coincides with the second component.

Write the dual vector space $\widehat{\mathfrak{h}}^{*}$ to the Cartan subalgebra in the form

$$
\widehat{\mathfrak{h}}^{*}=\mathfrak{h}^{*} \oplus \mathbb{C} \Lambda_{0} \oplus \mathbb{C} \delta,
$$

where

$$
\Lambda_{0}(\mathfrak{h} \oplus \mathbb{C} d)=0, \quad \delta(\mathfrak{h} \oplus \mathbb{C} K)=0, \quad \Lambda_{0}(K)=\delta(d)=1
$$

and $\nu(K)=\nu(d)=0$ for all $\nu \in \mathfrak{h}^{*}$. Let $\varepsilon_{1}, \ldots, \varepsilon_{m+n} \in \mathfrak{h}^{*}$ denote the basis of $\mathfrak{h}^{*}$ dual to the standard basis of $\mathfrak{h}$ so that $\varepsilon_{i}\left(e_{j j}\right)=\delta_{i j}$.

Given an element $\lambda \in \widehat{\mathfrak{h}}^{*}$, the Verma module $M(\lambda)$ with the highest weight $\lambda$ is defined as the induced module

$$
M(\lambda)=\mathrm{U}(\widehat{\mathfrak{g}}) \otimes_{\mathrm{U}(\widehat{\mathfrak{h}} \oplus \mathfrak{n})} \mathbb{C}_{\lambda},
$$


where $\mathbb{C}_{\lambda}$ is the one-dimensional $(\widehat{\mathfrak{h}} \oplus \widehat{\mathfrak{n}})$-module with the basis vector $1_{\lambda}$ such that

$$
\widehat{\mathfrak{n}} 1_{\lambda}=0 \quad \text { and } \quad h 1_{\lambda}=\lambda(h) 1_{\lambda}, \quad h \in \widehat{\mathfrak{h}} .
$$

We will identify $M(\lambda)$ with $\mathrm{U}\left(\widehat{\mathfrak{n}}_{-}\right)$as a vector space.

The highest weight $\lambda$ is at the critical level, if $\lambda(K)=n-m$. The vector space $\widehat{\mathfrak{h}}^{*}$ is equipped with the bilinear form $($, ) defined by

$$
\begin{aligned}
\left(\nu+a \Lambda_{0}+b \delta, \mu+a^{\prime} \Lambda_{0}+b^{\prime} \delta\right) & \\
& =\nu_{1} \mu_{1}+\cdots+\nu_{m} \mu_{m}-\nu_{m+1} \mu_{m+1}-\cdots-\nu_{m+n} \mu_{m+n}+a b^{\prime}+b a^{\prime}
\end{aligned}
$$

where $\nu=\nu_{1} \varepsilon_{1}+\cdots+\nu_{m+n} \varepsilon_{m+n}$ and $\mu=\mu_{1} \varepsilon_{1}+\cdots+\mu_{m+n} \varepsilon_{m+n}$. The condition that $\lambda$ is at the critical level can be written as $(\lambda+\rho, \delta)=0$, where the element $\rho \in \widehat{\mathfrak{h}}^{*}$ is defined by

$$
\rho=(m-1) \varepsilon_{1}+\cdots+\varepsilon_{m-1}-\varepsilon_{m+2}-\cdots-(n-1) \varepsilon_{m+n}+(m-n) \Lambda_{0} .
$$

The root system $\Delta \subset \widehat{\mathfrak{h}}^{*}$ of $\widehat{\mathfrak{g}}$ is formed by the elements $k \delta+\varepsilon_{i}-\varepsilon_{j}$ with $k \in \mathbb{Z}$ and $i \neq j$ and by the elements $k \delta$ with $k \in \mathbb{Z}, k \neq 0$. The real positive roots are given by

$$
\Delta_{r e}^{+}=\left\{k \delta+\varepsilon_{i}-\varepsilon_{j} \mid k>0, \quad i \neq j\right\} \cup\left\{\varepsilon_{i}-\varepsilon_{j} \mid i<j\right\} .
$$

An element $\lambda \in \widehat{\mathfrak{h}}^{*}$ is called a generic critical weight if $\lambda$ is at the critical level and

$$
(\lambda+\rho, \alpha) \neq \frac{p}{2}(\alpha, \alpha) \quad \text { for all } \quad p=1,2, \ldots \quad \text { and } \quad \alpha \in \Delta_{r e}^{+} .
$$

A singular vector of the Verma module is an element $v \in M(\lambda)$ such that $\widehat{\mathfrak{n}} v=0$. If $\lambda$ is a generic critical weight, then the subspace of singular vectors $M(\lambda)^{\widehat{\mathfrak{n}}}$ has the weight space decomposition of the form

$$
M(\lambda)^{\widehat{\mathfrak{n}}}=\bigoplus_{k \geqslant 0} M(\lambda)_{\lambda-k \delta}^{\widehat{\mathfrak{n}}}
$$

Moreover, $M(\lambda)^{\widehat{\mathfrak{n}}}$ possesses an algebra structure which is described in [17, Theorem 1.1]: the restriction of the projection $\mathcal{H} C$ defined in (3.18) to $M(\lambda)^{\widehat{\mathfrak{n}}}$ gives an algebra isomorphism

$$
\mathcal{H} C: M(\lambda)^{\widehat{\mathfrak{n}}} \cong \mathrm{U}\left(t^{-1} \mathfrak{h}\left[t^{-1}\right]\right)
$$

This result was applied in [17] to obtain the character formula for the simple quotient of $M(\lambda)$ thus proving the Kac-Kazhdan conjecture; see also an earlier paper [25] for another proof of this conjecture for affine Lie superalgebras of type $A(m-1 \mid n-1)$.

The next lemma will be needed for the proof of Theorem 3.10 below. For this lemma we regard $\lambda_{1}, \ldots, \lambda_{m+n}$ as independent variables. Consider the square matrix $\Lambda=\left[\Lambda_{k i}\right]$ 
with $1 \leqslant k, i \leqslant m+n$ whose entries are polynomials in the $\lambda_{i}$ and a complex parameter $r$ defined by the formulas

$$
\begin{aligned}
\Lambda_{k i}=\sum_{a+b+c=k-1} & h_{a}\left(\lambda_{m+1}+r+k-a, \ldots, \lambda_{m+n}+r+k-a\right) \\
& \times e_{b}\left(\lambda_{i+1}-r-c-1, \ldots, \lambda_{m}-r-c-1\right) e_{c}\left(\lambda_{1}, \ldots, \lambda_{i-1}\right)
\end{aligned}
$$

for $i=1, \ldots, m$, and

$$
\begin{array}{r}
\Lambda_{k i}=\sum_{a+b+c=k-1} h_{a}\left(\lambda_{i}+r+k-a, \ldots, \lambda_{m+n}+r+k-a\right) \\
\times h_{b}\left(\lambda_{m+1}+c, \ldots, \lambda_{i}+c\right) e_{c}\left(\lambda_{1}, \ldots, \lambda_{m}\right)
\end{array}
$$

for $i=m+1, \ldots, m+n$ with the summation taken over nonnegative integers $a, b, c$, and we used the following shifted versions of the complete and elementary symmetric polynomials:

$$
h_{a}\left(x_{1}, \ldots, x_{l}\right)=\sum_{l \geqslant i_{1} \geqslant \cdots \geqslant i_{a} \geqslant 1}\left(x_{i_{1}}+a-1\right) \ldots\left(x_{i_{a-1}}+1\right) x_{i_{a}}
$$

and

$$
e_{a}\left(x_{1}, \ldots, x_{l}\right)=\sum_{l>i_{1}>\cdots>i_{a}>1}\left(x_{i_{1}}-a+1\right) \ldots\left(x_{i_{a-1}}-1\right) x_{i_{a}} .
$$

Lemma 3.9. We have

$$
\begin{array}{r}
\operatorname{det} \Lambda=\prod_{1 \leqslant i<j \leqslant m}\left(\lambda_{i}-\lambda_{j}+j-i+r\right) \prod_{m+1 \leqslant i<j \leqslant m+n}\left(\lambda_{j}-\lambda_{i}-j+i-r\right) \\
\times \prod_{1 \leqslant i \leqslant m<j \leqslant m+n}\left(\lambda_{i}+\lambda_{j}-i-j+2 m+1\right) .
\end{array}
$$

Proof. Note that all entries in the top row of $\Lambda$ are equal to 1 . Then replacing the $i$-th column $\operatorname{col}_{i}$ of $\Lambda$ by the difference $\operatorname{col}_{i+1}-\operatorname{col}_{i}$ for $i=1, \ldots, m+n-1$ we find that $\operatorname{det} \Lambda$ equals the determinant of another square matrix of size $m+n-1$. All elements in each column of this matrix has a common linear factor. By taking out all these factors we are left with a matrix whose entries in the top row are equal to 1 and we can repeat the same procedure.

More precisely, define a sequence of matrices $\Lambda^{(s)}=\left[\Lambda_{k i}^{(s)}\right]$ with $s=0,1, \ldots, m+n-1$ so that $\Lambda^{(s)}$ is a square matrix of size $m+n-s$. We set $\Lambda^{(0)}=\Lambda$ and define the entries of $\Lambda^{(s)}$ inductively. For $s \geqslant 1$ we set

$$
\Lambda_{k i}^{(s)}=\frac{\Lambda_{k+1, i+1}^{(s-1)}-\Lambda_{k+1, i}^{(s-1)}}{\lambda_{i}-\lambda_{i+s}+s+r}
$$

if $1 \leqslant i \leqslant m-s$

$$
\Lambda_{k i}^{(s)}=\frac{\Lambda_{k+1, i+1}^{(s-1)}-\Lambda_{k+1, i}^{(s-1)}}{\lambda_{i}+\lambda_{i+s}-2 i-s+2 m+1}
$$


if $m-s+1 \leqslant i \leqslant m$, and

$$
\Lambda_{k i}^{(s)}=\frac{\Lambda_{k+1, i+1}^{(s-1)}-\Lambda_{k+1, i}^{(s-1)}}{\lambda_{i+s}-\lambda_{i}-s-r}
$$

if $m+1 \leqslant i \leqslant m+n-s$. It is straightforward to verify that these relations are satisfied by the matrix elements

$$
\begin{aligned}
\Lambda_{k i}^{(s)}=\sum_{a+b+c=k-1} & h_{a}\left(\lambda_{m+1}+r+k+s-a, \ldots, \lambda_{m+n}+r+k+s-a\right) \\
& \times e_{b}\left(\lambda_{i+s+1}-r-c-s-1, \ldots, \lambda_{m}-r-c-s-1\right) e_{c}\left(\lambda_{1}, \ldots, \lambda_{i-1}\right)
\end{aligned}
$$

for $1 \leqslant i \leqslant m-s$,

$$
\begin{aligned}
\Lambda_{k i}^{(s)}=\sum_{a+b+c=k-1} & h_{a}\left(\lambda_{m+1}+r+k+s-a, \ldots, \lambda_{m+n}+r+k+s-a\right) \\
& \times h_{b}\left(\lambda_{m+1}+c+m-i+1, \ldots, \lambda_{i+s}+c+m-i+1\right) e_{c}\left(\lambda_{1}, \ldots, \lambda_{i-1}\right)
\end{aligned}
$$

for $m-s+1 \leqslant i \leqslant m$ and

$$
\begin{gathered}
\Lambda_{k i}^{(s)}=\sum_{a+b+c=k-1} h_{a}\left(\lambda_{i}+r+k+s-a, \ldots, \lambda_{m+n}+r+k+s-a\right) \\
\times h_{b}\left(\lambda_{m+1}+c, \ldots, \lambda_{i+s}+c\right) e_{c}\left(\lambda_{1}, \ldots, \lambda_{m}\right)
\end{gathered}
$$

for $m+1 \leqslant i \leqslant m+n-s$. Indeed, if $1 \leqslant i \leqslant m-s$, then the difference $\Lambda_{k+1, i+1}^{(s-1)}-\Lambda_{k+1, i}^{(s-1)}$ can be found by calculating

$$
\begin{aligned}
\sum_{b+c=d} e_{b}\left(\lambda_{i+s+1}-r-c-s, \ldots, \lambda_{m}-r-c-s\right) e_{c}\left(\lambda_{1}, \ldots, \lambda_{i}\right) & \\
& -\sum_{b+c=d} e_{b}\left(\lambda_{i+s}-r-c-s, \ldots, \lambda_{m}-r-c-s\right) e_{c}\left(\lambda_{1}, \ldots, \lambda_{i-1}\right)
\end{aligned}
$$

with $d=k-a-1$. Using the recurrence relations

$$
e_{a}\left(x_{1}, \ldots, x_{l}\right)=e_{a}\left(x_{2}, \ldots, x_{l}\right)+e_{a-1}\left(x_{2}-1, \ldots, x_{l}-1\right) x_{1}
$$

and

$$
e_{a}\left(x_{1}, \ldots, x_{l}\right)=e_{a}\left(x_{1}, \ldots, x_{l-1}\right)+\left(x_{l}-a+1\right) e_{a-1}\left(x_{1}, \ldots, x_{l-1}\right)
$$

we bring the difference of the sums to the form

$$
\left(\lambda_{i}-\lambda_{i+s}+s+r\right) \sum_{b+c=d-1} e_{b}\left(\lambda_{i+s+1}-r-c-s-1, \ldots, \lambda_{m}-r-c-s-1\right) e_{c}\left(\lambda_{1}, \ldots, \lambda_{i-1}\right)
$$

thus verifying (3.23). Checking that the $\Lambda_{k i}^{(s)}$ satisfy (3.24) and (3.25) is quite similar by using analogous recurrence relations for the polynomials $h_{a}\left(x_{1}, \ldots, x_{l}\right)$ together with the relation

$$
h_{a}\left(x_{1}, \ldots, x_{l}\right)=h_{a}\left(x_{1}+1, \ldots, x_{l-1}+1\right)+\left(x_{l}-l+1\right) h_{a-1}\left(x_{1}+1, \ldots, x_{l}+1\right)
$$


which follows from the well-known formula for the generating series of these polynomials:

$$
1+\sum_{a=1}^{\infty} \frac{h_{a}\left(x_{1}, \ldots, x_{l}\right)}{(u+1) \ldots(u+a)}=\frac{u(u-1) \ldots(u-l+1)}{\left(u-x_{1}-l+1\right) \ldots\left(u-x_{l}\right)} .
$$

Since the determinant of the $1 \times 1$ matrix $\Lambda^{(m+n-1)}=[1]$ is equal to 1 , taking the product of the linear factors occurring in the denominators in (3.23), (3.24) and (3.25) for all values of $s$, we get the desired formula for $\operatorname{det} \Lambda$.

Now recall that the Fourier coefficients of all fields defined in Corollary 3.5 belong to the center of the local completion $\mathrm{U}_{n-m}\left(\widehat{\mathfrak{g l}}_{m \mid n}\right)_{\text {loc }}$ of the universal enveloping algebra $\mathrm{U}\left(\widehat{\mathfrak{g l}}_{m \mid n}\right)$. Each of these coefficients is a well-defined operator in the Verma module $M(\lambda)$ at the critical level, preserving the subspace $M(\lambda)^{\widehat{\mathfrak{n}}}$ of singular vectors. We now aim to produce algebraically independent generators of $M(\lambda)^{\widehat{\mathfrak{n}}}$ regarded as a commutative algebra for generic critical weights $\lambda$.

Recall the fields $\sigma_{k l}(z)$ defined in Corollary 3.5] and introduce their Fourier coefficients by the expansions

$$
\sigma_{k l}(z)=\sum_{r \in \mathbb{Z}} \sigma_{k l}[r] z^{-r-l}
$$

Then $\sigma_{k l}[r] 1_{\lambda}$ is a singular vector of $M(\lambda)$ of weight $\lambda+r \delta$ (this vector is zero for $r>0$ ).

Theorem 3.10. Suppose that $\lambda$ is a generic critical weight. Then

$$
M(\lambda)^{\widehat{\mathfrak{n}}}=\mathbb{C}\left[\sigma_{k k}[r] \mid k=1, \ldots, m+n, r<0\right] 1_{\lambda}
$$

so that any singular vector of $M(\lambda)$ can be expressed as a unique polynomial in the operators $\sigma_{k k}[r]$ with $k=1, \ldots, m+n$ and $r<0$ applied to the highest vector.

Proof. Denote by $\sigma_{k l}^{\lambda}[r]$ the image of the element $\sigma_{k l}[r] 1_{\lambda}$ under the isomorphism $\mathcal{H C}$ defined in (3.20). It will be sufficient to show that the elements $\sigma_{k k}^{\lambda}[r]$ with $k=1, \ldots, m+n$ and $r<0$ are algebraically independent generators of the algebra $\mathrm{U}\left(t^{-1} \mathfrak{h}\left[t^{-1}\right]\right)$. We will use the explicit formulas for the operators $\sigma_{k k}[r]$ implied by Proposition 2.3 . Keeping the same notation and applying the first relation of that proposition we get

$$
\begin{aligned}
: \operatorname{str}_{1, \ldots, k} A_{k} T_{1}(z) \ldots T_{k}(z): & =\sum_{I} \frac{1}{\alpha_{m+1} ! \ldots \alpha_{m+n} !} \sum_{\sigma \in \mathfrak{S}_{k}} \operatorname{sgn} \sigma \cdot \varphi(\sigma, \sigma I, I) \\
& \times:\left(\partial_{z}+\widehat{E}(z)\right)_{i_{\sigma(1)} i_{1}} \ldots\left(\partial_{z}+\widehat{E}(z)\right)_{i_{\sigma(k)} i_{k}}:(-1)^{\gamma(\sigma I, I)}
\end{aligned}
$$

Given $I$ and $\sigma$, use the definition of the normal ordering (3.4) and apply the corresponding summand to the highest vector:

$$
\begin{aligned}
& :\left(\partial_{z}+\widehat{E}(z)\right)_{i_{\sigma(1)} i_{1}} C(z): 1_{\lambda} \\
& \quad=\left(\delta_{i_{\sigma(1)} i_{1}} \partial_{z}+(-1)^{\bar{\tau}_{\sigma(1)}} e_{i_{\sigma(1)} i_{1}}(z)_{+}\right) C(z) 1_{\lambda}+(-1)^{\bar{\imath}_{1}} C(z) e_{i_{\sigma(1)} i_{1}}(z)_{-} 1_{\lambda}
\end{aligned}
$$


where we set

$$
C(z)=:\left(\partial_{z}+\widehat{E}(z)\right)_{i_{\sigma(2)} i_{2}} \ldots\left(\partial_{z}+\widehat{E}(z)\right)_{i_{\sigma(k)} i_{k}}:
$$

We have

$$
e_{i_{\sigma(1)} i_{1}}(z)_{-} 1_{\lambda}=\sum_{r \geqslant 0} z^{-r-1} e_{i_{\sigma(1)} i_{1}}[r] 1_{\lambda} .
$$

Since $i_{1} \geqslant \cdots \geqslant i_{l} \geqslant m+1>i_{l+1}>\cdots>i_{k}$, this expression is zero unless $i_{\sigma(1)}=i_{1}$. In the latter case it equals $z^{-1} \lambda_{i_{1}} 1_{\lambda}$, where the $\lambda_{i}$ are the components of the highest weight $\lambda=\lambda_{1} \varepsilon_{1}+\cdots+\lambda_{m+n} \varepsilon_{m+n}+(n-m) \Lambda_{0}+b \delta$ (the value of $b$ is irrelevant). On the other hand,

$$
e_{i_{\sigma(1)} i_{1}}(z)_{+} C(z) 1_{\lambda}=\sum_{r<0} z^{-r-1} e_{i_{\sigma(1)} i_{1}}[r] C(z) 1_{\lambda} .
$$

The coefficient of each power of $z$ belongs to the kernel of the projection (3.18) unless $i_{\sigma(1)}=i_{1}$. Proceeding by induction and using the same argument for $C(z) 1_{\lambda}$ we conclude that after the application to the highest weight vector $1_{\lambda}$, the summation in $(3.26)$ can be restricted to those permutations $\sigma$ which stabilize the multiset $I$ so that $\sigma I=I$. The number of such permutations is $\alpha_{m+1} ! \ldots \alpha_{m+n} !$. Furthermore, by (2.8) for any such $\sigma$ the $\operatorname{sign} \varphi(\sigma, I, I)$ coincides with $\operatorname{sgn} \sigma$ so that under the isomorphism $\mathcal{H C}$ defined in (3.20) we have

$$
\begin{aligned}
: \operatorname{str}_{1, \ldots, k} A_{k} T_{1}(z) & \ldots T_{k}(z): 1_{\lambda} \mapsto \\
\sum_{I}( & \left.-\partial_{z}+\lambda_{i_{1}} z^{-1}+e_{i_{1} i_{1}}(z)_{+}\right) \ldots\left(-\partial_{z}+\lambda_{i_{l}} z^{-1}+e_{i_{l} i_{l}}(z)_{+}\right) \\
& \times\left(\partial_{z}+\lambda_{i_{l+1}} z^{-1}+e_{i_{l+1} i_{l+1}}(z)_{+}\right) \ldots\left(\partial_{z}+\lambda_{i_{k}} z^{-1}+e_{i_{k} i_{k}}(z)_{+}\right)
\end{aligned}
$$

summed over multisets $I=\left\{i_{1} \geqslant \cdots \geqslant i_{l} \geqslant m+1>i_{l+1}>\cdots>i_{k}\right\}$ with $l=0, \ldots, k$. Thus the elements $\sigma_{k l}^{\lambda}[r] \in \mathrm{U}\left(t^{-1} \mathfrak{h}\left[t^{-1}\right]\right)$ are found by writing the differential operator in (3.28) in the form

$$
\sigma_{k 0}^{\lambda}(z) \partial_{z}^{k}+\sigma_{k 1}^{\lambda}(z) \partial_{z}^{k-1}+\cdots+\sigma_{k k}^{\lambda}(z)
$$

with

$$
\sigma_{k l}^{\lambda}(z)=\sum_{r \leqslant 0} \sigma_{k l}^{\lambda}[r] z^{-r-l}
$$

In particular,

$$
\sigma_{11}^{\lambda}[0]=\lambda_{1}+\cdots+\lambda_{m+n}, \quad \sigma_{11}^{\lambda}[r]=e_{11}[r]+\cdots+e_{m+n, m+n}[r], \quad r<0,
$$

and the elements $\sigma_{k k}^{\lambda}[r]$ satisfy the recurrence relations

$$
\sigma_{k k}^{\lambda}[r]=\widetilde{\sigma}_{k k}^{\lambda}[r]+\left(\lambda_{m+n}+r+k-1\right) \sigma_{k-1, k-1}^{\lambda}[r]+\sum_{p=r}^{-1} e_{m+n, m+n}[p] \sigma_{k-1, k-1}^{\lambda}[r-p]
$$


for $r<0$, and

$$
\sigma_{k k}^{\lambda}[0]=\widetilde{\sigma}_{k k}^{\lambda}[0]+\left(\lambda_{m+n}+k-1\right) \sigma_{k-1, k-1}^{\lambda}[0],
$$

where the elements $\widetilde{\sigma}_{k k}^{\lambda}[r]$ are defined by the expansion

$$
\begin{aligned}
& \sum_{I}\left(-\partial_{z}+\lambda_{i_{1}} z^{-1}+e_{i_{1} i_{1}}(z)_{+}\right) \ldots\left(-\partial_{z}+\lambda_{i_{l}} z^{-1}+e_{i_{l} i_{l}}(z)_{+}\right) \\
& \times\left(\partial_{z}+\lambda_{i_{l+1}} z^{-1}+e_{i_{l+1} i_{l+1}}(z)_{+}\right) \\
& \ldots\left(\partial_{z}+\lambda_{i_{k}} z^{-1}+e_{i_{k} i_{k}}(z)_{+}\right) \\
& \quad=\widetilde{\sigma}_{k 0}^{\lambda}(z) \partial_{z}^{k}+\widetilde{\sigma}_{k 1}^{\lambda}(z) \partial_{z}^{k-1}+\cdots+\widetilde{\sigma}_{k k}^{\lambda}(z)
\end{aligned}
$$

summed over multisets $I=\left\{m+n-1 \geqslant i_{1} \geqslant \cdots \geqslant i_{l} \geqslant m+1>i_{l+1}>\cdots>i_{k}\right\}$ with $l=0, \ldots, k$ (we assume that $n \geqslant 1$ as the calculation in the case $n=0$ is quite similar with some simplifications).

Observe that $\mathrm{U}\left(t^{-1} \mathfrak{h}\left[t^{-1}\right]\right)$ is a graded algebra with the degree of the generator $e_{i i}[r]$ with $r<0$ equal to $-r$. The above recurrence relations show that each element $\sigma_{k k}^{\lambda}[r]$ is homogeneous of degree $-r$. Therefore, in order to complete the proof of the theorem it will be sufficient to verify that each generator $e_{i i}[r]$ can be expressed as a polynomial in the elements $\sigma_{k k}^{\lambda}[r]$. The algebraic independence of these elements will then follow from the fact that the homogeneous subspaces of $\mathrm{U}\left(t^{-1} \mathfrak{h}\left[t^{-1}\right]\right)$ are finite-dimensional.

We will use the reverse induction on $r$ and suppose that $r \leqslant-1$. Using the above recurrence relations we can write $\sigma_{k k}^{\lambda}[r]$ as a homogeneous polynomial of degree $-r$ in the generators $e_{i i}[p]$. By the induction hypothesis, all generators $e_{i i}[p]$ with $p>r$ can be expressed as polynomials in the $\sigma_{k k}^{\lambda}[q]$. Furthermore, modulo polynomials in those generators, each element $\sigma_{k k}^{\lambda}[r]$ with $k=1, \ldots, m+n$ is given as a linear combination of the generators $e_{i i}[r]$ with $i=1, \ldots, m+n$. Thus, we only need to show that the corresponding matrix is nonsingular for any $r$.

As we only need to keep linear terms in the recurrence relations, they are simplified to

$$
\sigma_{k k}^{\lambda}[r]=\widetilde{\sigma}_{k k}^{\lambda}[r]+\left(\lambda_{m+n}+r+k-1\right) \sigma_{k-1, k-1}^{\lambda}[r]+e_{m+n, m+n}[r] \sigma_{k-1, k-1}^{\lambda}[0]
$$

for $r<0$. Using this together with (3.30), we derive that the desired linear combinations take the form

$$
\sigma_{k k}^{\lambda}[r]=\sum_{i=1}^{m+n} \Lambda_{k i} e_{i i}[r], \quad k=1, \ldots, m+n,
$$

where the coefficients $\Lambda_{k i}$ are given by (3.21) and (3.22). The determinant of the matrix $\Lambda=\left[\Lambda_{k i}\right]$ is found in Lemma 3.9. Recalling that $\lambda$ is a generic critical weight and applying (3.19) for the positive real roots of the form $\alpha=k \delta+\varepsilon_{i}-\varepsilon_{j}$ with $1 \leqslant i<j \leqslant m$ we find that $\lambda_{i}-\lambda_{j}+j-i+r \neq 0$ for all $r<0$. Similarly, taking $\alpha=k \delta+\varepsilon_{j}-\varepsilon_{i}$ with $m+1 \leqslant i<j \leqslant m+n$ and $\alpha=k \delta+\varepsilon_{i}-\varepsilon_{j}$ with $1 \leqslant i \leqslant m<j \leqslant m+n$ we conclude that for the respective values of $i$ and $j$ we have $\lambda_{j}-\lambda_{i}-j+i-r \neq 0$ for all $r<0$ 
and $\lambda_{i}+\lambda_{j}-i-j+2 m+1 \neq 0$. Hence, the determinant of the matrix is nonzero. This completes the proof.

Now consider the fields $h_{k l}(z)$ defined in Corollary 3.5. Explicit formulas for these fields are found by using the second relation in Proposition 2.3 which implies the identity

$$
\begin{aligned}
: \operatorname{str}_{1, \ldots, k} H_{k} T_{1}(z) \ldots T_{k}(z): & =\sum_{I} \frac{1}{\alpha_{1} ! \ldots \alpha_{m} !} \sum_{\sigma \in \mathfrak{S}_{k}} \psi(\sigma, I, \sigma I) \\
& \times:\left(\partial_{z}+\widehat{E}(z)\right)_{i_{1} i_{\sigma(1)}} \ldots\left(\partial_{z}+\widehat{E}(z)\right)_{i_{k} i_{\sigma(k)}}:(-1)^{\gamma(I, \sigma I)},
\end{aligned}
$$

where we keep the notation of that proposition. Introduce the Fourier coefficients of the fields $h_{k l}(z)$ by the expansions

$$
h_{k l}(z)=\sum_{r \in \mathbb{Z}} h_{k l}[r] z^{-r-l}
$$

Corollary 3.11. Suppose that $\lambda$ is a generic critical weight. Then

$$
M(\lambda)^{\widehat{\mathfrak{n}}}=\mathbb{C}\left[h_{k k}[r] \mid k=1, \ldots, m+n, r<0\right] 1_{\lambda}
$$

so that any singular vector of $M(\lambda)$ can be expressed as a unique polynomial in the operators $h_{k k}[r]$ with $k=1, \ldots, m+n$ and $r<0$ applied to the highest vector.

Proof. Let $h_{k l}^{\lambda}[r]$ denote the image of $h_{k l}[r] 1_{\lambda}$ under the isomorphism (3.20). As in the proof of Theorem 3.10 , we will show that the elements $h_{k k}^{\lambda}[r]$ with $k=1, \ldots, m+n$ and $r<0$ are algebraically independent generators of the algebra $\mathrm{U}\left(t^{-1} \mathfrak{h}\left[t^{-1}\right]\right)$.

Using generating series, we can write relations (3.28) in the form

$$
\begin{aligned}
& \sum_{k=0}^{\infty} u^{k}: \operatorname{str}_{1, \ldots, k} A_{k} T_{1}(z) \ldots T_{k}(z): 1_{\lambda} \mapsto \\
& \left(1+u\left(\partial_{z}-\lambda_{m+n} z^{-1}-e_{m+n, m+n}(z)_{+}\right)\right)^{-1} \ldots\left(1+u\left(\partial_{z}-\lambda_{m+1} z^{-1}-e_{m+1, m+1}(z)_{+}\right)\right)^{-1} \\
& \quad \times\left(1+u\left(\partial_{z}+\lambda_{m} z^{-1}+e_{m m}(z)_{+}\right)\right) \ldots\left(1+u\left(\partial_{z}+\lambda_{1} z^{-1}+e_{11}(z)_{+}\right)\right) .
\end{aligned}
$$

Hence, by Theorem 2.2 ,

$$
\begin{aligned}
& \sum_{k=0}^{\infty} u^{k}: \operatorname{str}_{1, \ldots, k} H_{k} T_{1}(z) \ldots T_{k}(z): 1_{\lambda} \mapsto \\
& \quad\left(1-u\left(\partial_{z}+\lambda_{1} z^{-1}+e_{11}(z)_{+}\right)\right)^{-1} \ldots\left(1-u\left(\partial_{z}+\lambda_{m} z^{-1}+e_{m m}(z)_{+}\right)\right)^{-1} \\
& \quad \times\left(1+u\left(-\partial_{z}+\lambda_{m+1} z^{-1}+e_{m+1, m+1}(z)_{+}\right)\right) \ldots\left(1+u\left(-\partial_{z}+\lambda_{1} z^{-1}+e_{m+n, m+n}(z)_{+}\right)\right) .
\end{aligned}
$$


Therefore, taking the coefficient of $u^{k}$ we come to the relation

$$
\begin{aligned}
& h_{k 0}^{\lambda}(z) \partial_{z}^{k}+h_{k 1}^{\lambda}(z) \partial_{z}^{k-1}+\cdots+h_{k k}^{\lambda}(z) \\
& =\sum_{I}\left(\partial_{z}+\lambda_{i_{1}} z^{-1}+e_{i_{1} i_{1}}(z)_{+}\right) \ldots\left(\partial_{z}+\lambda_{i_{l}} z^{-1}+e_{i_{l} i_{l}}(z)_{+}\right) \\
& \quad \times\left(-\partial_{z}+\lambda_{i_{l+1}} z^{-1}+e_{i_{l+1} i_{l+1}}(z)_{+}\right) \ldots\left(-\partial_{z}+\lambda_{i_{k}} z^{-1}+e_{i_{k} i_{k}}(z)_{+}\right)
\end{aligned}
$$

summed over multisets $I=\left\{i_{1} \leqslant \cdots \leqslant i_{l} \leqslant m<i_{l+1}<\cdots<i_{k}\right\}$ with $l=0, \ldots, k$. In particular, we have

$$
h_{11}^{\lambda}[0]=\lambda_{1}+\cdots+\lambda_{m+n}, \quad h_{11}^{\lambda}[r]=e_{11}[r]+\cdots+e_{m+n, m+n}[r], \quad r<0,
$$

and the elements $h_{k k}^{\lambda}[r]$ satisfy the recurrence relations

$$
h_{k k}^{\lambda}[r]=\widetilde{h}_{k k}^{\lambda}[r]+\left(\lambda_{1}-r-k+1\right) h_{k-1, k-1}^{\lambda}[r]+\sum_{p=r}^{-1} e_{11}[p] h_{k-1, k-1}^{\lambda}[r-p]
$$

for $r<0$, and

$$
h_{k k}^{\lambda}[0]=\widetilde{h}_{k k}^{\lambda}[0]+\left(\lambda_{1}-k+1\right) h_{k-1, k-1}^{\lambda}[0],
$$

where $\widetilde{h}_{k k}^{\lambda}[r]$ are the elements of the algebra $\mathrm{U}\left(t^{-1} \mathfrak{h}_{m-1 \mid n}\left[t^{-1}\right]\right)$ defined as the coefficients in the expansion of the corresponding differential operator associated with the natural subalgebra $\mathfrak{h}_{m-1 \mid n} \subset \mathfrak{h}$ generated by the elements $e_{i i}[r]$ with $2 \leqslant i \leqslant m+n$. Arguing as in the proof of Theorem 3.10, we will keep only linear terms in the expansion of $h_{k k}^{\lambda}[r]$ so that the recurrence relations simplify to

$$
h_{k k}^{\lambda}[r]=\widetilde{h}_{k k}^{\lambda}[r]+\left(\lambda_{1}-r-k+1\right) h_{k-1, k-1}^{\lambda}[r]+e_{11}[r] h_{k-1, k-1}^{\lambda}[0]
$$

for $r<0$. Observe now that if we change the notation by setting $\lambda_{i}:=-\lambda_{m+n-i+1}$, $e_{i i}[r]:=e_{m+n-i+1, m+n-i+1}[r]$ for $r<0$, and

$$
\sigma_{k k}^{\lambda}[r]:= \begin{cases}(-1)^{k+1} h_{k k}^{\lambda}[r] & \text { if } \quad r<0 \\ (-1)^{k} h_{k k}^{\lambda}[r] & \text { if } \quad r=0\end{cases}
$$

then the recurrence relations would take exactly the same form as those in the proof of Theorem 3.10 for the elements $\sigma_{k k}^{\lambda^{\prime}}[r]$ associated with the Lie superalgebra $\widehat{\mathfrak{g l}}_{n \mid m}$ and the highest weight

$$
\lambda^{\prime}=\left(-\lambda_{m+n}, \ldots,-\lambda_{m+1},-\lambda_{m}, \ldots,-\lambda_{1}\right) .
$$

It remains to verify that the corresponding determinant of Lemma 3.9] does not vanish under the specialization at $\lambda^{\prime}$. This value is easily found and it turns out to be given by the same product as in Lemma 3.9, with each linear factor replaced by its negative. It was checked in the proof of Theorem 3.10 that each factor is nonzero. 
Applying the Newton identity (2.32), we can also calculate the images under the isomorphism $\mathcal{H C}$ of the singular vectors of the Verma module $M(\lambda)$ associated with the normally ordered traces of powers of the matrix $T(z)$; see Corollary 3.5. Using the notation introduced in the proofs of Theorem 3.10 and Corollary 3.11 set

$$
h_{k}^{\lambda}(z)=h_{k 0}^{\lambda}(z) \partial_{z}^{k}+h_{k 1}^{\lambda}(z) \partial_{z}^{k-1}+\cdots+h_{k k}^{\lambda}(z)
$$

and

$$
\sigma_{k}^{\lambda}(z)=\sigma_{k 0}^{\lambda}(z) \partial_{z}^{k}+\sigma_{k 1}^{\lambda}(z) \partial_{z}^{k-1}+\cdots+\sigma_{k k}^{\lambda}(z) .
$$

Denote by $s_{k l}^{\lambda}(z)$ the image of the series $s_{k l}(z) 1_{\lambda}$ under the isomorphism $\mathcal{H} C$ defined in (3.20), so that

$$
: \operatorname{str} T(z)^{k}: 1_{\lambda}=s_{k 0}^{\lambda}(z) \partial_{z}^{k}+s_{k 1}^{\lambda}(z) \partial_{z}^{k-1}+\cdots+s_{k k}^{\lambda}(z) .
$$

Corollary 3.12. The series $s_{k l}^{\lambda}(z)$ can be found from the relations

$$
s_{k 0}^{\lambda}(z) \partial_{z}^{k}+s_{k 1}^{\lambda}(z) \partial_{z}^{k-1}+\cdots+s_{k k}^{\lambda}(z)=\sum_{l=0}^{k-1}(-1)^{l}(l+1) h_{k-l-1}^{\lambda}(z) \sigma_{l+1}^{\lambda}(z) .
$$

Proof. This is immediate from Theorem 2.13,

\section{References}

[1] F. A. Berezin, Introduction to superanalysis, Mathematical Physics and Applied Mathematics, vol. 9. D. Reidel Publishing Co., Dordrecht, 1987. Edited and with a foreword by A. A. Kirillov. With an appendix by V. I. Ogievetsky. Translated from the Russian by J. Niederle and R. Kotecký. Translation edited by D. Leites.

[2] S. Caracciolo, A. D. Sokal and A. Sportiello, Noncommutative determinants, CauchyBinet formulae, and Capelli-type identities. I. Generalizations of the Capelli and Turnbull identities, Electron. J. Combin. 16 (2009), no. 1, Research Paper 103, 43 pp.

[3] A. Chervov and G. Falqui, Manin matrices and Talalaev's formula, J. Phys. A: Math. Theor. 41 (2008), 194006 (28pp).

[4] A. Chervov, G. Falqui and V. Rubtsov, Algebraic properties of Manin matrices 1, Adv. Appl. Math. 43 (2009), 239-315.

[5] A. V. Chervov and A. I. Molev, On higher order Sugawara operators, Int. Math. Res. Not. (2009), no. 9, 1612-1635. 
[6] A. Chervov and D. Talalaev, Quantum spectral curves, quantum integrable systems and the geometric Langlands correspondence, preprint arXiv:hep-th/0604128.

[7] B. Feigin and E. Frenkel, Affine Kac-Moody algebras at the critical level and GelfandDikii algebras, Int. J. Mod. Phys. A7, Suppl. 1A (1992), 197-215.

[8] B. Feigin, E. Frenkel and N. Reshetikhin, Gaudin model, Bethe ansatz and critical level, Comm. Math. Phys. 166 (1994), 27-62.

[9] D. Foata and G.-N. Han, A new proof of the Garoufalidis-Lê-Zeilberger quantum MacMahon Master Theorem, J. Algebra 307 (2007), 424-431.

[10] D. Foata and G.-N. Han, A basis for the right quantum algebra and the " $1=q$ " principle, J. Algebraic Combin. 27 (2008), 163-172.

[11] E. Frenkel, Wakimoto modules, opers and the center at the critical level, Adv. Math. 195 (2005), 297-404.

[12] E. Frenkel and D. Ben-Zvi, Vertex algebras and algebraic curves, Mathematical Surveys and Monographs, 88. American Mathematical Society, Providence, RI, 2001.

[13] S. Garoufalidis, Thang T. Q. Lê and D. Zeilberger, The quantum MacMahon master theorem, Proc. Nat. Acad. Sci. USA 103 (2006), 13928-13931.

[14] I. Gelfand, S. Gelfand, V. Retakh and R. Wilson, Quasideterminants, Adv. Math. 193 (2005), 56-141.

[15] I. M. Gelfand, D. Krob, A. Lascoux, B. Leclerc, V. S. Retakh and J.-Y. Thibon, Noncommutative symmetric functions, Adv. Math. 112 (1995), 218-348.

[16] R. Goodman and N. Wallach, Higher-order Sugawara operators for affine Lie algebras, Trans. Amer. Math. Soc. 315 (1989), 1-55.

[17] M. Gorelik, On a generic Verma module at the critical level over affine Lie superalgebras, Int. Math. Res. Not. (2007), Article 014, 41 pp.

[18] L. Gow, On the Yangian $Y\left(\mathfrak{g l}_{m \mid n}\right)$ and its quantum Berezinian, Czech. J. Phys. 55 (2005), 1415-1420.

[19] L. Gow, Gauss decomposition of the Yangian $Y\left(\mathfrak{g l}_{m \mid n}\right)$, Comm. Math. Phys. 276 (2007), 799-825.

[20] D. Gurevich, P. Pyatov and P. Saponov, Spectral parameterization for the power sums of quantum supermatrix, preprint arXiv:0805.0177. 
[21] P. H. Hai, B. Kriegk and M. Lorenz, N-homogeneous superalgebras, J. Noncommut. Geom. 2 (2008), 1-51.

[22] P. H. Hai and M. Lorenz, Koszul algebras and the quantum MacMahon master theorem, Bull. London Math. Soc. 277 (2007), 667-676.

[23] T. Hayashi, Sugawara operators and Kac-Kazhdan conjecture, Invent. Math. 94 (1988), 13-52.

[24] A. Isaev, O. Ogievetsky and P. Pyatov, Generalized Cayley-Hamilton-Newton identities, Czechoslovak J. Phys. 48 (1998), 1369-1374.

[25] K. Iohara and Y. Koga, Wakimoto modules for the affine Lie superalgebras $A(m-1 \mid n-1)^{(1)}$ and $D(2,1, a)^{(1)}$, Math. Proc. Camb. Phil. Soc. 132 (2002), 419-433.

[26] V. Kac, Vertex algebras for beginners, University Lecture Series, 10. American Mathematical Society, Providence, RI, 1997.

[27] H. M. Khudaverdian and Th. Th. Voronov, Berezinians, exterior powers and recurrent sequences, Lett. Math. Phys. 74 (2005), 201-228.

[28] M. Konvalinka and I. Pak, Non-commutative extensions of the MacMahon Master Theorem, Adv. Math. 216 (2007), 29-61.

[29] P. P. Kulish and N. Manojlovic, Creation operators and Bethe vectors of the osp $(1 \mid 2)$ Gaudin model, J. Math. Phys. 42 (2001), 4757-4778.

[30] Yu. I. Manin, Some remarks on Koszul algebras and quantum groups, Ann. Inst. Fourier (Grenoble) 37 (1987), 191-205.

[31] Yu. I. Manin, Quantum groups and noncommutative geometry, Université de Montréal, Centre de Recherches Mathématiques, Montréal, QC, 1988.

[32] A. Molev, Yangians and classical Lie algebras, Mathematical Surveys and Monographs, 143. American Mathematical Society, Providence, RI, 2007.

[33] A. I. Molev, Feigin-Frenkel center in types B, C and D, Invent. Math. 191 (2013), $1-34$.

[34] A. I. Molev and E. E. Mukhin, Yangian characters and classical $\mathcal{W}$-algebras, preprint arXiv:1212.4032.

[35] E. Mukhin, V. Tarasov and A. Varchenko, Bethe eigenvectors of higher transfer matrices, J. Stat. Mech. Theory Exp. 2006, no. 8, P08002, 44 pp. 
[36] E. Mukhin, V. Tarasov and A. Varchenko, Bethe algebra of Gaudin model, CalogeroMoser space and Cherednik algebra, preprint arXiv:0906.5185.

[37] M. L. Nazarov, Quantum Berezinian and the classical Capelli identity, Lett. Math. Phys. 21 (1991), 123-131.

[38] Th. Schmitt, Some identities for Berezin's function, in: "Seminar analysis", 1981/82, pp. 146-161. Akad. Wiss. DDR, Berlin (1982).

[39] D. V. Talalaev, The quantum Gaudin system, Funct. Anal. Appl. 40 (2006), 73-77. 